\title{
ASYMPTOTIC HYPERFUNCTIONS, TEMPERED HYPERFUNCTIONS, AND ASYMPTOTIC EXPANSIONS
}

ANDREAS U. SCHMIDT

Received 20 September 2004

We introduce new subclasses of Fourier hyperfunctions of mixed type, satisfying polynomial growth conditions at infinity, and develop their sheaf and duality theory. We use Fourier transformation and duality to examine relations of these asymptotic and tempered hyperfunctions to known classes of test functions and distributions, especially the Gel'fand-Shilov spaces. Further it is shown that the asymptotic hyperfunctions, which decay faster than any negative power, are precisely the class that allows asymptotic expansions at infinity. These asymptotic expansions are carried over to the higher-dimensional case by applying the Radon transformation for hyperfunctions.

\section{Introduction}

Since the advent of Sato's hyperfunctions [48, 49], and the introduction of Fourier hyperfunctions by Kawai [29], the research field of hyperfunctions has become grossly diversified. Main branches are the algebro-analytic [28] and the functional analytic approach to the subject. Within the latter, in which the present study takes its place, a large number of special classes of hyperfunctions has been considered (cf. the introductions of $[22,23,47])$. The construction of two new subclasses of Fourier hyperfunctions in this paper is driven by two motives: firstly, their relation to known classes of distributions and hyperfunctions, and, secondly yet not less, their intended application. The two classes of tempered, respectively, asymptotic hyperfunctions that we consider, satisfy two extreme cases of polynomial bounds at infinity. The latter fall off faster than any power, while the former are allowed to grow as an arbitrary finite power. With respect to the first motive above, we show that tempered and asymptotic hyperfunctions fit into and extend the scheme of generalized functions introduced by Gel'fand and Shilov [15]. In this way, we gain insight in the operations of duality and Fourier transform on our and several other spaces of test and generalized functions, paralleling earlier studies $[17,42,55]$. The second motive has two roots: the application of hyperfunctions in theoretical physics, and the more general and classical subject of asymptotic expansions $[41,63,64]$. For the first, there is a long standing view that in a fundamental formulation of quantum field theory, the mathematical problems of QFT can be seen as a problem 
of the choice of the "right" class of generalized functions for the representation of quantum fields $[2,24,33,51,50,56,57,58,65]$. Among other developments, this has led to formulations of QFT in terms of ultradistributions $[8,9,46]$ and finally hyperfunctions $[2,3,36,37,38,39,40]$. Furthermore, there are results which relate these problems, and especially the most difficult subject of renormalization [59], to asymptotic expansions $[4,5,62]$. Also, infrared divergences show a connection to these [53]. This altogether inspired our interest in the possibility of asymptotic expansions for a suitable class of hyperfunctions, and we are able to show that our asymptotic hyperfunctions are well suited in this respect.

This paper contains some of the essential parts of [52] in Sections 3 and 4, and is organized as follows.

In Section 2, we establish the sheaf theory of tempered and asymptotic hyperfunctions of general type with values in a Fréchet space by the duality method. The strategy follows coarsely the proceeding of [22] and uses methods and arguments from other sources, see, for example, $[34,45]$, which are almost classical. Therefore, to omit superfluous repetitions and in order to clarify the line of argument, we state only the core results, postponing all proofs to Appendix A. A further generalization to tempered and asymptotic hyperfunctions with values in a general Hilbert space as in [23] seems possible, but we do not undertake this.

It should be noted at this point that in the one-dimensional case, the sheaf theory for tempered and asymptotic hyperfunctions can be built upon relatively elementary complex-analytic methods, as in the case of ordinary hyperfunctions, see $[26,32]$. In essence, this amounts to analogies of Runge's approximation theorem and Mittag-Leffler's theorem with polynomial growth conditions. This is done in [52], where polynomial bounds at infinity for hyperfunctions in one dimension are established. For the duality theory of these hyperfunctions in one dimension, it is useful to follow the spirit of the famous Phragmén-Lindelöf principle, to obtain polynomial bounds on integrals along a contour around an unbounded domain in $\mathbb{C}$ from bounds in the interior. This result of separate interest is contained in [54].

Section 3 explores the functional analytic structure of the spaces of tempered and asymptotic hyperfunctions. To that end, we combine duality to test function spaces with behavior under Fourier transformation. We are able to show the identity of tempered hyperfunctions to the dual of the Gel'fand-Shilov space $\mathscr{S}^{1}$, see [15]. This way, we extend the Gel'fand-Shilov scheme of test function and distribution spaces by hyperfunctions with polynomial growth conditions.

Section 4 contains an application of asymptotic hyperfunctions which we regard as essential. We use them to extend the asymptotic expansions of distributions exhibited in $[12,13]$ to hyperfunctions (cf. also the related results for ultradistributions in [7]). It turns out that the asymptotic ones are the natural objects in the category of hyperfunctions for such expansions. We start by exploring the one-dimensional case. Generalization to higher dimensions could trivially be done using Cartesian products, but we prefer a more symmetric approach which uses the Radon transformation for hyperfunctions described by Takiguchi and Kaneko in [60].

Finally, the appendix contains the proofs of the statements in Section 2. 


\section{Hyperfunctions by the duality method}

At the heart of the duality method lies a general theorem on the existence and uniqueness of a flabby sheaf of Fréchet spaces under quite weak conditions which are streamlined for the use with duals of appropriate test function spaces. It first appeared in [25] and was further generalized in [22]. We use a slightly weaker statement, which is sufficient for our needs.

Theorem 2.1 (Shapira-Junker-Ito, see [22, Theorem 1.2.1]). Take X to be a locally compact, $\sigma$-compact topological space satisfying the second axiom of countability. Assume there is a collection $\left\{F_{K}\right\}$ of Fréchet spaces labeled by the compact subsets $K$ of $X$ such that $F_{\varnothing}=0$ and for any two compacta $K_{1}, K_{2} \subset X$ the following hold:

(i) if $K_{1} \subset K_{2}$, then there exists a continuous injection $i_{K_{1}, K_{2}}: F_{K_{1}} \rightarrow F_{K_{2}}$,

(ii) if $K_{1} \subset K_{2}$ is such that every connected component of $K_{2}$ intersects $K_{1}$, then $i_{K_{1}, K_{2}}$ has dense image,

(iii) the sequence of Fréchet spaces

$$
0 \longrightarrow F_{K_{1} \cap K_{2}} \longrightarrow F_{K_{1}} \oplus F_{K_{2}} \stackrel{\lambda}{\rightarrow} F_{K_{1} \cup K_{2}} \longrightarrow 0
$$

with $\lambda:\left(u_{1}, u_{2}\right) \mapsto u_{1}-u_{2}$, is an exact topological sequence,

(iv) for every at most countable family $\left\{K_{i}\right\}$ of compacta in $X$ holds $F_{K}=\bigcap_{i} F_{K_{i}}$, where $K=\bigcap_{i} K_{i}$.

Then, there exists exactly one flabby sheaf $\mathscr{F}$ on $X$ with $\Gamma_{K}(X, \mathscr{F})=F_{K}$ for every compact set $K$ in $X$.

In practice, the spaces $F_{K}$ will be spaces of locally analytic functionals on real subsets of $\mathbb{C}^{n}$. To obtain all types of Fourier hyperfunctions, the base space $X$ is set out as a combination of $\mathbb{R}^{n}$ and two types of radial compactifications of $\mathbb{R}^{n}$ : as usual we denote by $\mathbb{D}^{n}, n \in \mathbb{N}$, the radial compactification of $\mathbb{R}^{n}$ in the sense of Kawai, see, for example, [26]. To denote the base spaces on which germs of holomorphic functions, respectively, hyperfunctions of arbitrary mixed type live, we use triple indices of nonnegative integers $\mathbf{n} \stackrel{\text { def }}{=}\left(n_{1}, n_{2}, n_{3}\right), \mathbf{n} \in \llbracket$. Here, we denote by $\llbracket$ the subset of $\mathbf{n} \in \mathbb{N}_{0}^{3}$ such that $|\mathbf{n}| \stackrel{\text { def }}{=} n_{1}+$ $n_{2}+n_{3} \neq 0$. With this, we set $\mathbb{Q}^{\mathbf{n}} \stackrel{\text { def }}{=} \mathbb{C}^{n_{1}} \times(\mathbb{D}+i \mathbb{R})^{n_{2}} \times(\mathbb{D} \times i \mathbb{D})^{n_{3}}$, for $\mathbf{n} \in \mathbb{0}$. Here, the real subspace $\mathbb{D}^{\mathbf{n}} \stackrel{\text { def }}{=} \mathbb{R}^{n_{1}} \times\left(\mathbb{D}^{1}\right)^{n_{2}} \times\left(\mathbb{D}^{1}\right)^{n_{3}}$ is conceived as a compact subset of $\mathbb{Q}^{\mathbf{n}}$. We will later introduce separate symbols for the common special cases of indices $\mathbf{n}$ corresponding to ordinary, Fourier-, modified-, and mixed-type hyperfunctions. The reader will find it easy to reconstruct the notation of [22, Section 2.1] from ours. We set $z=\left(z^{\prime}, z^{\prime \prime}, z^{\prime \prime \prime}\right)$ for $z \in \mathbb{C}^{|\mathbf{n}|}$, with $z^{\prime}=\left(z_{1}, \ldots, z_{n_{1}}\right), z^{\prime \prime}=\left(z_{n_{1}+1}, \ldots, z_{n_{1}+n_{2}}\right), z^{\prime \prime \prime}=\left(z_{n_{1}+n_{2}+1}, \ldots, z_{|\mathbf{n}|}\right)$. For any $S \subset \mathbb{Q}^{\mathbf{n}}$ we write $S_{\mathbb{C}^{|\mathbf{n}|}}$ for $S \cap \mathbb{C}^{|\mathbf{n}|}$. We denote by $\overline{\bar{U}}$ the closure of $U$ in $\mathbb{Q}^{\mathbf{n}}$ and by $K^{\circ}$ the interior of $K$.

Definition 2.2. For an open set $U \subset \mathbb{Q}^{\mathbf{n}}$ let $O_{\infty}(U)$ (resp., $O_{-\infty}(U)$ ) be the space of all holomorphic functions $f$ on $U_{\mathbb{C}|\mathbf{n}|}$ such that for any compact set $K \subset U$, there exists a 
$\gamma \in \mathbb{R}($ resp., for all $\gamma \in \mathbb{R})$ and

$$
\sup _{K_{\mathbb{C}|\mathbf{n}|}}\left|f(z)\left(1+\left|z^{\prime \prime}\right|+\left|z^{\prime \prime \prime}\right|\right)^{-\gamma}\right|<\infty
$$

holds. The sheaves $\mathrm{O}_{ \pm \infty}$ of germs of tempered, respectively, asymptotic holomorphic functions are the sheafifications of the presheaves generated by the spaces $O_{ \pm \infty}(U)$ of local sections.

Next we introduce topologies on the spaces of local sections.

Definition 2.3. Let $K \subset \mathbb{Q}^{\mathbf{n}}$ be compact and $U \subset \mathbb{Q}^{\mathbf{n}}$ be open. For $m \in \mathbb{Z}$ and a compact set $K \subset \mathbb{Q}^{\mathbf{n}}$, set

$$
{\overline{\|f\|_{m, K}}}_{m} \stackrel{\text { def }}{=} \sup _{K_{\mathbb{C}|\mathbf{n}|}}\left|f(z)\left(1+\left|z^{\prime \prime}\right|+\left|z^{\prime \prime \prime}\right|\right)^{-m}\right|
$$

whenever this makes sense for a function $f$. Denote by $\overline{\mathrm{O}}_{m}^{B}(K)$ the space of holomorphic functions $f$ on $K_{\mathbb{C}^{|\mathbf{n}|}}^{\circ}$, which are continuous on $K_{\mathbb{C}|\mathbf{n}|}$, and such that $\|f\|_{m, K}<\infty$ holds. Choose a fundamental system $\left\{V_{m}\right\}$ of neighborhoods of $K$ with $V_{m+1} \Subset V_{m}$ and a sequence $\left\{L_{m}\right\}$ of compacta which exhausts $U$. Set

$$
\begin{aligned}
& \mathrm{O}_{\infty}(K) \stackrel{\text { def }}{=} \underline{\lim }_{\overline{0}_{m}^{B}}\left(\overline{\overline{V_{m}}}\right), \quad \mathrm{O}_{-\infty}(U) \stackrel{\text { def }}{=} \underline{\lim }_{\longleftarrow} \overline{0}_{-m}^{B}\left(L_{m}\right), \\
& \mathrm{O}_{\infty}(U) \stackrel{\text { def }}{=} \mathrm{lim}_{\infty} \mathrm{O}_{\infty}\left(L_{m}\right), \quad \mathrm{O}_{-\infty}(K) \stackrel{\text { def }}{=} \underline{\lim } \widehat{O}_{-\infty}\left(V_{m}\right) \text {, }
\end{aligned}
$$

thereby introducing locally convex topologies on these spaces.

Proposition 2.4. The spaces $\mathrm{O}_{ \pm \infty}(K)$ are DFS-spaces and $\mathrm{O}_{ \pm \infty}(U)$ are FS-spaces. All these spaces are nuclear.

The sheaves of germs of tempered, respectively, asymptotic real analytic functions are defined by $\mathcal{P}_{ \pm \infty} \stackrel{\text { def }}{=} \mathcal{O}_{ \pm \infty} \mid \mathbb{D}^{\mathrm{n}}$. The spaces of sections $\mathcal{P}_{ \pm \infty}(K)$ of $\mathcal{P}_{ \pm \infty}$ on a compact set $K \subset \mathbb{D}^{\mathbf{n}}$ are the DFS-spaces $\mathrm{O}_{ \pm \infty}(K)$. The spaces of local sections of the sheaves $\mathrm{O}_{ \pm \infty}$ and $\mathcal{P}_{ \pm \infty}$ exhibit the usual tensor product decomposition property.

Proposition 2.5. For compact sets $K \subset \mathbb{Q}^{\mathbf{n}}$ and $L \subset \mathbb{Q}^{\mathbf{m}}$, the topological isomorphisms are

(i) $\hat{O}_{ \pm \infty}(U \times V) \cong \hat{O}_{ \pm \infty}(U) \hat{\otimes} \hat{O}_{ \pm \infty}(V), U \subset \mathbb{Q}^{\mathbf{n}}, V \subset \mathbb{Q}^{\mathbf{m}}$ open,

(ii) $\mathrm{O}_{ \pm \infty}(K \times L) \cong \mathrm{O}_{ \pm \infty}(K) \hat{\otimes} \mathcal{O}_{ \pm \infty}(L), K \subset \mathbb{Q}^{\mathbf{n}}, L \subset \mathbb{Q}^{\mathbf{m}}$ compact,

(iii) $\mathcal{P}_{ \pm \infty}(K \times L) \cong \mathcal{P}_{ \pm \infty}(K) \hat{\otimes} \mathcal{P}_{ \pm \infty}(L), K \subset \mathbb{D}^{\mathbf{n}}, L \subset \mathbb{D}^{\mathbf{m}}$ compact,

(iv) $\mathcal{P}_{ \pm \infty}\left(\mathbb{Q}^{\left(n_{1}, n_{2}, n_{3}\right)}\right) \cong \mathscr{A}\left(\mathbb{R}^{n_{1}}\right) \hat{\otimes} \mathcal{P}_{ \pm \infty}\left(\mathbb{D}^{\left(0, n_{2}, n_{3}\right)}\right)$,

where $\mathscr{A}\left(\mathbb{R}^{n}\right)$ denotes the space of ordinary real analytic functions on $\mathbb{R}^{n}$.

By duality, one could derive Schwartz-type kernel theorems for the tempered and asymptotic hyperfunctions to be defined below from the above proposition, as in [22, Section 3.1] or [6], but we will be content with leaving this issue on the level of test functions.

Theorem 2.6. For every compact set $K \subset \mathbb{D}^{\mathbf{n}}$ holds $\mathrm{H}_{K}^{1}\left(\mathbb{D}^{\mathbf{n}} ; \mathcal{P}_{ \pm \infty}\right)=0$. 
This theorem is the basis for the localization of hyperfunctions. Namely, by considering the long exact sequence of cohomology groups

$$
\begin{aligned}
0 \longrightarrow \mathcal{P}_{ \pm \infty}\left(K_{1} \cup K_{2}\right) \longrightarrow \mathcal{P}_{ \pm \infty}\left(K_{1}\right) \oplus \mathcal{P}_{ \pm \infty}\left(K_{2}\right) \longrightarrow \mathcal{P}_{ \pm \infty}\left(K_{1} \cap K_{2}\right) \\
\longrightarrow \mathrm{H}_{K_{1} \cup K_{2}}^{1}\left(\mathbb{D}^{\mathbf{n}} ; \mathcal{P}_{ \pm \infty}\right) \longrightarrow \cdots
\end{aligned}
$$

for two compact sets $K_{1}, K_{2} \subset \mathbb{D}^{\mathbf{n}}$, we immediately derive from it the following important conclusion, which is dual to condition (iii) of Theorem 2.1.

Corollary 2.7. The following sequence is exact:

$$
0 \longrightarrow \mathcal{P}_{ \pm \infty}\left(K_{1} \cup K_{2}\right) \longrightarrow \mathcal{P}_{ \pm \infty}\left(K_{1}\right) \oplus \mathcal{P}_{ \pm \infty}\left(K_{2}\right) \longrightarrow \mathcal{P}_{ \pm \infty}\left(K_{1} \cap K_{2}\right) \longrightarrow 0
$$

The last main ingredient is an approximation theorem of Runge type.

Theorem 2.8. $\mathcal{P}_{ \pm \infty}\left(\mathbb{D}^{\mathbf{n}}\right)$ is dense in $\mathcal{P}_{ \pm \infty}(K)$ for $K \subset \mathbb{D}^{\mathbf{n}}$ compact.

Now let $E$ be any Fréchet space. For an open set $U \subset \mathbb{Q}^{\mathbf{n}}$ we call the spaces $O_{ \pm \infty}^{\prime}(U ; E) \stackrel{\text { def }}{=}$ $L\left(O_{ \pm \infty}(U) ; E\right)$ of all continuous linear mappings from $O_{ \pm \infty}(U)$ into $E$ the asymptotic, respectively, tempered analytic functionals on $U$ with values in E. Similarly, we define ${O_{ \pm \infty}^{\prime}}^{\prime}(K ; E) \stackrel{\text { def }}{=} L\left(O_{ \pm \infty}(K) ; E\right)$, respectively, $\mathcal{P}_{ \pm \infty}^{\prime}(K ; E) \stackrel{\text { def }}{=} L\left(O_{ \pm \infty}(K) ; E\right)$ for $K \subset \mathbb{Q}^{\mathbf{n}}$, respectively, $K \subset \mathbb{D}^{\mathbf{n}}$ compact. All these spaces are endowed with the topology of convergence on compact subsets. Then, by virtue of [61, Proposition 50.5], see also [35, proof of Theorem 5.7], we have the following.

Proposition 2.9. For any Fréchet space E, the following hold:

(i) $O_{ \pm \infty}^{\prime}(U ; E) \cong O_{ \pm \infty}^{\prime}(U) \hat{\otimes} E$, for $U \subset \mathbb{Q}^{\mathbf{n}}$ open,

(ii) $O_{ \pm \infty}^{\prime}(K ; E) \cong O_{ \pm \infty}^{\prime}(K) \hat{\otimes} E$, for $K \subset \mathbb{Q}^{\mathbf{n}}$ compact,

(iii) $\mathcal{P}_{ \pm \infty}^{\prime}(K ; E) \cong \mathcal{P}_{ \pm \infty}^{\prime}(K) \hat{\otimes} E$, for $K \subset \mathbb{D}^{\mathbf{n}}$ compact.

We say that a compact set $K \subset U \subset \mathbb{Q}^{\mathbf{n}}$ is a carrier for a section $F \in O_{ \pm \infty}^{\prime}(U ; E)$ if $F$ can be extended to an element of $\sigma_{ \pm \infty}^{\prime}(K ; E)$. The functional $F$ is said to be carried by an open subset $V$ in $U$ if it is carried by some compact subset of $V$. If a compact set $K$ in $U \subset \mathbb{D}^{\text {n }}$ has the Runge property, and thus $\mathcal{P}_{ \pm \infty}(U)$ is dense in $\mathcal{P}_{ \pm \infty}(K)$ by Theorem 2.8 , then $F$ is carried by $K$ if and only if it is carried by all open neighborhoods of $K$ in $U$.

By using the dual of the exact sequence of Corollary 2.7, and by induction, we easily see that $\bigcap_{i} \mathcal{P}_{ \pm \infty}^{\prime}\left(K_{i} ; E\right)=\mathcal{P}_{ \pm \infty}^{\prime}\left(\bigcap_{i} K_{i} ; E\right)$ for every countable family $\left\{K_{i}\right\}$ of compacta in $\mathbb{D}^{\mathbf{n}}$. Then, Zorn's lemma implies that for every functional $F \in \mathcal{P}_{ \pm \infty}^{\prime}\left(\mathbb{D}^{\mathbf{n}} ; E\right)$ with $F \neq 0$, we can find a smallest compact set $K$ in $\mathbb{D}^{\mathbf{n}}$ which is a carrier for $F$. We call $K$ the support of $F$ and denote it by $\operatorname{supp}(F)$ (cf. [22, Theorems 2.3.4 and 2.3.5]). Then, the identity $\mathcal{P}_{ \pm \infty}^{\prime}\left(K_{i} ; E\right)=\left\{F \in \mathcal{P}_{ \pm \infty}^{\prime}\left(\mathbb{D}^{\mathbf{n}} ; E\right) \mid \operatorname{supp}(F) \subset K\right\}$ easily follows.

With these preparations, we are ready to define the sheaves of tempered and asymptotic hyperfunctions of general type with values in a Fréchet space E. Namely, the mapping $K \mapsto \mathcal{P}_{ \pm \infty}^{\prime}(K ; E)$, which assigns a Fréchet space to every compact set $K \subset \mathbb{D}^{\mathbf{n}}$, satisfies all conditions of the Shapira-Junker-Ito Theorem 2.1 (cf. [22, proof of Theorem 2.4.1]). Thus we have the following. 
THEOREM 2.10. There exists exactly one flabby sheaf ${ }^{E} \mathcal{Q}_{ \pm \infty}$ such that for every compact set $K \subset \mathbb{D}^{\mathbf{n}}$ holds $\Gamma_{K}\left(\mathbb{D}^{\mathbf{n}},{ }^{E} \mathcal{Q}_{ \pm \infty}\right)=\mathcal{P}_{\mp \infty}^{\prime}(K ; E)$.

There is a natural embedding of flabby sheaves

$$
{ }^{E} \mathcal{Q}_{-\infty} \hookrightarrow{ }^{E} \mathcal{Q}_{\infty} \hookrightarrow{ }^{E} \mathcal{Q}
$$

of asymptotic into tempered into ordinary Fourier hyperfunctions on $\mathbb{D}^{\mathbf{n}}$, induced by the continuous inclusions of the respective test functions spaces.

\section{The structure of tempered and asymptotic hyperfunctions}

In this section, we specialize to the case of scalar-valued, unmodified, tempered, and asymptotic hyperfunctions, that is, we consider $\mathcal{Q}_{ \pm \infty}=\mathbb{C}_{\mathcal{Q}_{ \pm \infty}}$ on $\mathbb{D}^{n}=\mathbb{D}^{(0, n, 0)} \subset \mathbb{Q}^{n}=$ $\mathbb{Q}^{(0, n, 0)}$. The following theorem establishes the orthantic boundary value representation for global sections of these sheaves (cf. [26, Section 7.1]). We do not go into developing a duality theory for local sections, resembling Poincarè-Serre duality for cohomology groups, but rather present duality theorem which relates globally defined tempered and asymptotic hyperfunctions to boundary values of holomorphic functions with the same growth or decay behavior.

Theorem 3.1. There is a linear, topological isomorphism

$$
\mathcal{Q}_{ \pm \infty}\left(\mathbb{D}^{n}\right) \cong \mathbb{O}_{ \pm \infty}\left(W \# \mathbb{D}^{n}\right) / \sum_{j=1}^{n} \mathbb{O}_{ \pm \infty}\left(W \#_{j} \mathbb{D}^{n}\right)
$$

for every open, cylindrical neighborhood $W$ of $\mathbb{D}^{n}$ in $\mathbb{Q}^{n}$.

Here, for $W=W_{1} \times \cdots \times W_{n}$ and a compact, cylindrical subset $K=K_{1} \times \cdots \times K_{n}$ of $W$ we define

$$
\begin{gathered}
W \# K \stackrel{\text { def }}{=}\left(W_{1} \backslash K_{1}\right) \times \cdots \times\left(W_{n} \backslash K_{n}\right), \\
W \#_{j} K \stackrel{\text { def }}{=}\left(W_{1} \backslash K_{1}\right) \times \cdots \times \underbrace{W_{j}}_{\text {Omitted }} \times \cdots \times\left(W_{n} \backslash K_{n}\right) .
\end{gathered}
$$

We will not give a detailed proof of this theorem, since the existing ones for Fourier hyperfunctions can be literally applied in our case, see, for example, the clear exposition in [2, Part C]. We nevertheless comment on the essential points. For an equivalence class $[F]$ in one of the quotients defined above and a function $f \in \mathcal{P}_{\mp \infty}\left(\mathbb{D}^{n}\right)$ one defines an inner product

$$
\langle[F], f\rangle=-\int_{\Gamma_{1}} \cdots \int_{\Gamma_{n}} F\left(z_{1}, \ldots, z_{n}\right) f\left(z_{1}, \ldots, z_{n}\right) \mathrm{d} z_{1} \cdots \mathrm{d} z_{n}
$$

where the integration plane $\Gamma=\Gamma_{1} \times \cdots \times \Gamma_{n}$ has to be chosen to lie in the common domain of holomorphy of $F$ and $f$. Since it is of the form of a Cartesian product, Cauchy's 
theorem ensures independence of the bilinear form of the special choice of $\Gamma$. One easily sees that the linear functional $T_{[F]}=\langle[F], \cdot\rangle$ is continuous. That the mapping $F \mapsto T_{[F]}$ is injective is essentially an application of Cauchy's integral formula, but with an exponentially decaying kernel. This kernel

$$
h_{z}(w) \stackrel{\text { def }}{=} \prod_{i=1}^{n} \frac{-1}{2 \pi i} \cdot \frac{\mathrm{e}^{-\left(z_{i}-w_{i}\right)^{2}}}{z_{i}-w_{i}}
$$

is also used to show surjectivity of $T$ by evaluating it on a given functional $T \in \mathcal{Q}_{ \pm \infty}\left(\mathbb{D}^{n}\right)$. The function $T\left(h_{z}\right)$ is in $0_{ \pm \infty}\left(W \# \mathbb{D}^{n}\right)$, since $h$ preserves the present asymptotic, respectively, tempered growth condition as can easily be verified by explicit estimation, see [52], and defines $T$ via $T_{\left[T\left(h_{z}\right)\right]}=T$ and accordingly, $T\left(h_{z}\right)$ is called a defining function for the hyperfunction $T$.

The set $W \# \mathbb{D}^{n}$ decomposes into $2^{n}$ connected components labeled by the signs $\sigma=$ $\left(\sigma_{1}, \ldots, \sigma_{n}\right)$ of the imaginary parts of the components $\left(z_{1}, \ldots, z_{n}\right)$ of the coordinate $z$. By this decomposition, every tempered or asymptotic hyperfunction possesses the orthantic boundary value representation

$$
f(x)=\sum_{\sigma} F_{\sigma}\left(x+i \Gamma_{\sigma} 0\right), \quad F_{\sigma}(z) \in \mathrm{O}_{ \pm \infty}\left(\mathbb{D}^{n}+i \Gamma_{\sigma} 0\right)
$$

where $\Gamma_{\sigma} \stackrel{\text { def }}{=}\left\{x \in \mathbb{R}^{n} \mid \sigma \cdot x>0\right\}$ is the $\sigma$ th orthant and $F_{\sigma}$ is holomorphic on an infinitesimal wedge of type $\mathbb{D}^{n}+i \Gamma_{\sigma} 0$, see [26, page 82$]$. Contact with representations by boundary values from other infinitesimal wedges can be made by convolution of a hyperfunction $f \in \mathbb{Q}_{ \pm \infty}\left(\mathbb{D}^{n}\right)$ with the exponentially decreasing Radon decomposition kernel $W_{*}(x, \omega)$, $\omega \in S^{n-1}$, which preserves the polynomial growth conditions on $f$, see [60, Appendix]. Consistency of all such representations is assured by Martineau's edge of the wedge theorem with polynomial decay conditions, which we may cite now in a form suitable for tempered and asymptotic hyperfunctions.

Theorem 3.2 [60, Theorem A.6]. Let $f(x)$ be a Fourier hyperfunction with a set of defining functions $\left\{F_{j}(z) \in \mathcal{O}_{ \pm \infty}\left(\mathbb{D}^{n}+i \Gamma_{j} 0\right)\right\}_{j=1}^{N}$. Assume $f=0$ in $\mathcal{Q}_{ \pm \infty}\left(\mathbb{D}^{n}\right)$. Then for any choice of proper subcones $\Gamma_{j}^{\prime} \Subset \Gamma_{j}$ there exist wedge-analytic functions $F_{j k} \in O_{ \pm \infty}\left(\mathbb{D}^{n}+i\left(\Gamma_{j}^{\prime}+\Gamma_{k}^{\prime}\right) 0\right)$ such that

$$
F_{j k}=-F_{k j}, \quad F_{j}(z)=\sum_{k=1}^{N} F_{i k}(z)
$$

on an infinitesimal wedge of type $\mathbb{D}^{n}+i \Gamma_{j} 0$.

The Fourier transformation $\mathscr{F}$ on $\mathcal{Q}_{ \pm \infty}\left(\mathbb{D}^{n}\right)$, see [26, Chapter 7], can be defined as usual by taking the boundary value of the Fourier-Laplace transformation of a single boundary value and extending linearly to the formal sums representing $f \in \mathcal{Q}_{ \pm \infty}\left(\mathbb{D}^{n}\right)$. It is consistent with the embedding $\mathcal{Q}_{ \pm \infty}\left(\mathbb{D}^{n}\right) \hookrightarrow \mathcal{Q}\left(\mathbb{D}^{n}\right)$ (since the boundary value representations are), and the Fourier inversion formula holds. 
We first consider the Fourier transform of the space $\mathcal{P}_{-\infty}\left(\mathbb{D}^{n}\right)$ of asymptotic real analytic functions, which is the test function space of $\mathcal{Q}_{\infty}\left(\mathbb{D}^{n}\right)$. As one would expect, $\mathscr{F} \mathcal{P}_{-\infty}\left(\mathbb{D}^{n}\right)$ is a space of exponentially decreasing $C^{\infty}$-functions. Closer examination shows that it is one of the spaces $\beta_{\alpha}^{\beta}$ introduced by Gel'fand and Shilov. We give an equivalent definition of it.

Definition 3.3 [15, Chapter IV, Section 3]. Set

$$
\S_{1}\left(\mathbb{R}^{n}\right) \stackrel{\text { def }}{=}\left\{f \in C^{\infty}\left(\mathbb{R}^{n}\right)\left|\exists \delta>0, \forall \alpha: \sup _{x}\right| D_{x}^{\alpha} f(x) \mid \mathrm{e}^{\delta|x|}<\infty\right\} .
$$

The topology of $\delta_{1}\left(\mathbb{R}^{n}\right)$ is that of an inductive limit

$$
\wp_{1}\left(\mathbb{R}^{n}\right)=\underset{A \rightarrow \infty}{\lim } \wp_{1, A}\left(\mathbb{R}^{n}\right)
$$

of countably normed spaces, where $\delta_{1, A}\left(\mathbb{R}^{n}\right)$ is the space of all infinitely differentiable functions $f$ for which all the norms

$$
\|f\|_{S_{1} ; m, p} \stackrel{\text { def }}{=} \sup _{x,|\alpha| \leq p}\left|D_{x}^{\alpha} f(x)\right| \mathrm{e}^{m^{-1}\left(1-p^{-1}\right)|x|}
$$

are finite, where $m=\mathrm{e} A$, and $p=2,3, \ldots$

Theorem 3.4. The Fourier transformation $\mathscr{F}_{\mathscr{F}}: \mathcal{P}_{-\infty}\left(\mathbb{D}^{n}\right) \stackrel{\sim}{\rightarrow} \wp_{1}\left(\mathbb{R}^{n}\right)$ induces a linear topological isomorphism.

Proof. We choose an equivalent representation of the space $\mathcal{P}_{-\infty}\left(\mathbb{D}^{n}\right)$ as an inductive limit of countably normed spaces:

$$
\mathcal{P}_{-\infty}\left(\mathbb{D}^{n}\right) \cong \underset{m}{\lim } \overline{0}_{-\infty}^{B}\left(\overline{\overline{U_{m}}}\right) \quad \text { with } \overline{0}_{-\infty}^{B}\left(\overline{\overline{U_{m}}}\right) \stackrel{\text { def }}{=} \underbrace{\lim }_{k} \overline{0}_{-k}^{B}\left(\overline{\overline{U_{m}}}\right) .
$$

Here we use the special system $U_{m}=\mathbb{D}^{n}+i\{|\operatorname{Im} z|<1 / m\}$ of neighborhoods of $\mathbb{D}^{n}$. Now let $f \in \overline{\bar{O}}_{-\infty}^{B}\left(\overline{\overline{U_{m}}}\right)$ for an $m \in \mathbb{N}$. Since $f$ is an asymptotic function on the whole domain $\overline{\overline{U_{m}}} \mathbb{C}^{n}$, we can use Cauchy's theorem and dominated convergence to calculate its Fourier transform $\hat{f}$ by shifting the integration plane as follows:

$$
D_{\xi}^{\alpha} \hat{f}(\xi)=\int_{z=\mathbb{R}^{n}+i y}(-i z)^{\alpha} \mathrm{e}^{-i z \xi} f(z) \mathrm{d} z
$$


with arbitrary $|y| \leq 1 / m$. Choosing $z=x \mp i / m$ for $\xi \gtrless 0$, we estimate

$$
\begin{aligned}
\left|D_{\xi}^{\alpha} \hat{f}(\xi)\right| & \leq\|f\|_{-|\alpha|-n-1, \overline{\overline{U_{m}}}} \cdot\left|\int_{\mathbb{R}^{n}} \mathrm{e}^{-i x \xi} \mathrm{e}^{-|\xi| / m}(1+|x|)^{-n-1} \mathrm{~d} x\right| \\
& \leq C_{n} \cdot\|f\|_{-|\alpha|-n-1, \overline{\overline{U_{m}}}} \cdot \mathrm{e}^{-|\xi| / m},
\end{aligned}
$$

with certain $C_{n}>0$. This shows that $\hat{f}$ is an exponentially decreasing $C^{\infty}$-function, that is, an element of $\S_{1}\left(\mathbb{R}^{n}\right)$. Thus, for the norm $\|\cdot\|_{\delta_{1} ; m, p}$ on $\S_{1, A}\left(\mathbb{R}^{n}\right)$ with $A=m /$ e, we have

$$
\begin{aligned}
\|\hat{f}\|_{8_{1} ; m, p} & \leq C \cdot\|f\|_{-p-n-1, \overline{\overline{U_{m}}}} \cdot \sup _{\xi} \mathrm{e}^{-|\xi| / m} \mathrm{e}^{\left(1-p^{-1}\right)|\xi| / m} \\
& \leq C \cdot\|f\|_{-p-n-1, \overline{\overline{U_{m}}}} \cdot \sup _{\xi} \mathrm{e}^{-|\xi| /(m p)} \leq C \cdot\|f\|_{-p-n-1, \overline{\overline{U_{m}}}},
\end{aligned}
$$

and similarly for every $m^{\prime}>m$, which shows continuity of $\mathscr{F}$ with respect to the inductive limit topologies of $\mathcal{P}_{-\infty}\left(\mathbb{D}^{n}\right)$ and $\ell_{1}\left(\mathbb{R}^{n}\right)$. Then, by the classical Fourier inversion formula, $\mathscr{F}$ is a continuous linear bijection and the continuity of $\mathscr{F}^{-1}$ follows similarly as above.

Since the Fourier transformation acts on the Gel'fand-Shilov spaces by exchanging the indices, we can immediately place $\mathcal{P}_{-\infty}\left(\mathbb{D}^{n}\right)$ itself into the $\oint_{\alpha}^{\beta}$-scheme. Tempered hyperfunctions also find their place, since $\mathcal{P}_{-\infty}\left(\mathbb{D}^{n}\right)$ are their test functions.

Corollary 3.5. The space $\mathcal{P}_{-\infty}\left(\mathbb{D}^{n}\right)$ is topologically isomorphic to the function space $\mathcal{s}^{1}\left(\mathbb{R}^{n}\right)$ and $\mathcal{Q}_{\infty}\left(\mathbb{D}^{n}\right)$ is topologically isomorphic to the space $f^{1^{\prime}}\left(\mathbb{R}^{n}\right)$. The Fourier transformation induces a mapping $\mathscr{F}: \mathcal{Q}_{\infty}\left(\mathbb{D}^{n}\right) \stackrel{\sim}{\rightarrow} \wp_{1}^{\prime}\left(\mathbb{R}^{n}\right)$, which is a linear, topological isomorphism.

Modeled after the scheme exhibited above, we can now examine the Fourier transform of asymptotic hyperfunctions. It is by now clear that $\mathscr{F} \mathcal{Q}_{-\infty}\left(\mathbb{D}^{n}\right)$ is a space of smooth functions. They exhibit the infraexponential growth property which is typical for Fourier transforms of Fourier hyperfunctions.

Definition 3.6. Denote by $C^{\infty *}\left(\mathbb{R}^{n}\right)$ the space of infraexponential, smooth functions. These are all $f \in C^{\infty}\left(\mathbb{R}^{n}\right)$ such that for all $k \in \mathbb{N}$ and $\varepsilon>0$ exists a constant $C_{k, \varepsilon}>0$ with

$$
\left|\frac{\partial^{\alpha} f(x)}{\partial x^{\alpha}}\right| \leq C_{k, \varepsilon} \mathrm{e}^{\varepsilon|\operatorname{Re} z|}
$$

for all multi-indices $\alpha \in \mathbb{N}^{n}$ with $|\alpha| \leq k$. Equip $C^{\infty *}\left(\mathbb{R}^{n}\right)$ with the topology of a countably normed space induced by the norms

$$
\|f\|_{C^{\infty * *} ; m, p} \stackrel{\text { def }}{=} \sup _{x,|\alpha| \leq p}\left|D_{x}^{\alpha} f(x)\right| \mathrm{e}^{-|x| / m}
$$

for all $p, m \in \mathbb{N}$, and consider $C^{\infty *}\left(\mathbb{R}^{n}\right)$ to be completed in this topology. 
We note aside that with this topology, $C^{\infty *}$ is isomorphic to the test function space which was denoted by $\mathscr{P}$ in [12].

Theorem 3.7. The Fourier transformation $\mathscr{F}: \mathcal{Q}_{-\infty}\left(\mathbb{D}^{n}\right) \stackrel{\sim}{\rightarrow} C^{\infty} *\left(\mathbb{R}^{n}\right)$ induces a linear topological isomorphism.

Proof. Since $\mathscr{F}$ extends linearly to sums of boundary values, it suffices to consider an asymptotic hyperfunction $f$ represented by a single boundary value $f(x)=F\left(x+i \Gamma_{\sigma} 0\right)$ from an orthant $\Gamma_{\sigma}$. So, let $W$ be an infinitesimal wedge of type $\mathbb{D}^{n}+i \Gamma_{\sigma} 0$ and $F(x+i y) \in$ $\mathrm{O}_{-\infty}(W)$ be a defining function for $f$. Then, for every compact set $L \subset \mathbb{R}^{n}$ such that $K=\mathbb{D}^{n}+i L$ is compact in $W$, the estimate

$$
\begin{aligned}
\left|D_{\xi}^{\alpha} \hat{f}(\xi)\right| & =\left|\int_{\operatorname{Im} z=y}(-i z)^{\alpha} \mathrm{e}^{-i z \xi} F(z) \mathrm{d} z\right| \\
& \leq\|F\|_{-|\alpha|-n-1, K}\left|\int_{\mathbb{R}^{n}} \mathrm{e}^{-i x \xi} \mathrm{e}^{y \xi}(1+|x|)^{-n-1} \mathrm{~d} x\right|
\end{aligned}
$$

holds with arbitrary $y \in L$. This shows $\mathscr{F} f \in C^{\infty *}\left(\mathbb{R}^{n}\right)$ since $|y|$ can be made arbitrarily small, leaving the integral unchanged by Cauchy's theorem. On the other hand, let $\hat{G}(\xi) \in$ $C^{\infty *}\left(\mathbb{R}^{n}\right)$ be such that all derivatives of $\hat{G}$ decrease exponentially outside the closed cone $\overline{\Gamma_{\sigma}}$, which can be achieved by eventually decomposing the original function utilizing exponentially decreasing multipliers. Then, the inverse Fourier transform $G=\mathscr{F}^{-1} \widehat{G}$ is a boundary value $G(z) \in \mathbb{O}^{*}\left(\mathbb{D}^{n}+i \Gamma_{\sigma} 0\right)$ and thus a Fourier hyperfunction (cf. [26, Proposition 8.3.2]). It is an easy calculation to show $G(z)=O\left(|\operatorname{Re} z|^{-\infty}\right)$ locally uniformly in $\operatorname{Im} z$. This shows $\mathscr{F}^{-1} \hat{G} \in \mathcal{Q}_{-\infty}\left(\mathbb{D}^{n}\right)$. The inversion formula for Fourier hyperfunctions, see $\left[26\right.$, Theorem 8.3 .4 ], thus implies that $\mathscr{F}_{\mathcal{F}}$ is a linear bijection from $\mathcal{Q}_{-\infty}\left(\mathbb{D}^{n}\right)$ onto $C^{\infty *}\left(\mathbb{R}^{n}\right)$ with inverse $\mathscr{F}^{-1}$. It remains to show continuity. If we choose a special exhausting sequence of compacta $\left\{K_{j}=\mathbb{D}^{n}+i L_{j}\right\}_{j \in \mathbb{N}}$ for $W$, such that some points of the cylindrical surface $\{|\operatorname{Im} z|=|y|=1 / j\}$ are contained in $K_{j \mathbb{C}^{n}}$, then we can make $y$ in estimate (3.16) small enough to conclude

$$
\left|D_{\xi}^{\alpha} \hat{f}(\xi)\right| \leq C \cdot\|F\|_{-|\alpha|-n-1, K_{j}} \cdot \mathrm{e}^{|\xi| / j} .
$$

This yields

$$
\|\hat{f}\|_{C^{\infty *} ; m, p} \leq C \cdot\|F\|_{-p-n-1, K_{j}} \sup _{\xi} \mathrm{e}^{\left(j^{-1}-m^{-1}\right)|\xi|} \leq C \cdot\|F\|_{-p-n-1, K_{j}}
$$

for all $j \geq m$, showing continuity of $\mathscr{F}$ in the topologies of $\mathcal{Q}_{-\infty}\left(\mathbb{D}^{n}\right)$ and $C^{\infty *}\left(\mathbb{R}^{n}\right)$.

Again, we can immediately draw the following conclusion.

COROLlary 3.8. The Fourier transformation $\mathscr{F}_{\mathcal{F}}: \mathcal{P}_{\infty}\left(\mathbb{D}^{n}\right) \stackrel{\sim}{\rightarrow} C^{\infty *^{\prime}}\left(\mathbb{R}^{n}\right)$ induces a linear topological isomorphism.

The test function spaces $\varsigma_{\alpha}^{\beta}$ for $\alpha, \beta \geq 1$, are ordered in the Gel'fand-Shilov scheme according to two characteristics. Growth order, controlled by the lower index $\alpha$, which ranges from exponential decay for $\alpha=1$ to rapid (asymptotic in our terminology) decay 


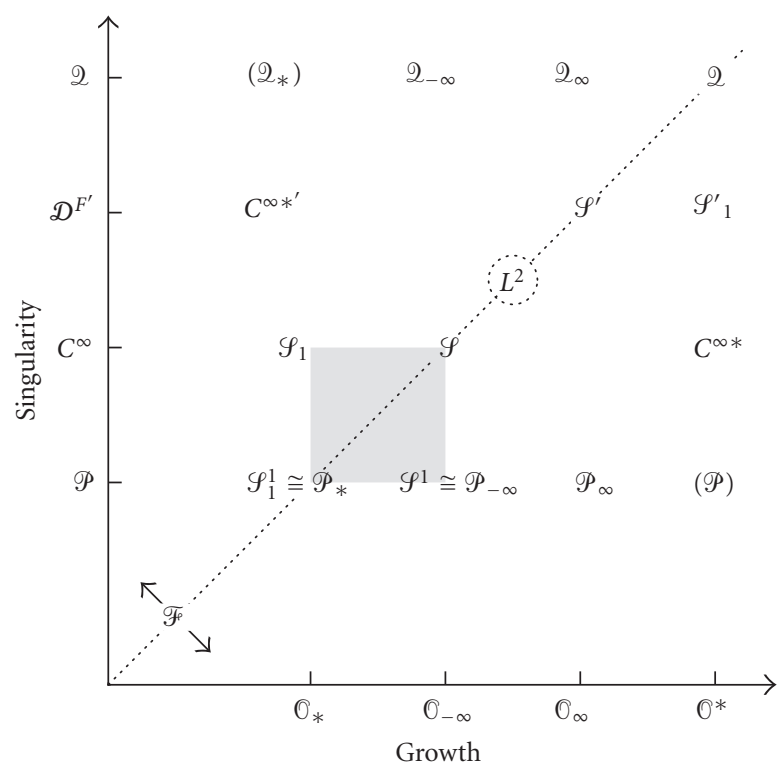

Figure 3.1. A diagram of generalized functions.

for $\alpha=\infty$. And regularity, which is that of real analytic functions in strip-like neighborhoods of the real axis for $\beta=1$, that is, the typical regularity of test function spaces of Fourier hyperfunctions, and on the other hand simple $C^{\infty}$-functions for $\beta=\infty$ (of course satisfying the growth conditions demanded by $\alpha$ to all derivatives). Note that, for example, the Schwartz space $\delta$ is nothing but $\delta_{\infty}^{\infty}$, and it is long known, see [36, Proposition 2.1], that $f_{1}^{1}\left(\mathbb{R}^{n}\right)$ is exactly the space $\mathcal{P}_{*}\left(\mathbb{D}^{n}\right)$ of exponentially decaying, real analytic test functions whose dual is the space $\mathbb{Q}\left(\mathbb{D}^{n}\right)$ of Fourier hyperfunctions.

We can use our two Paley-Wiener-type Theorems 3.4 and 3.7 and their corollaries to extend this scheme largely to include asymptotic, tempered, and Fourier hyperfunctions. The result is shown in Figure 3.1. On the ordinate are marked four types of growth conditions: exponential decay, asymptotic, that is, rapid decay, tempered growth, and infraexponential growth, symbolized in that order by $\mathrm{O}_{*}, \mathrm{O}_{-\infty}, \mathrm{O}_{\infty}, \mathrm{O}^{*}$. Here, the use of the symbol for holomorphic functions is justified by the embedding of the various spaces into spaces of hyperfunctions with the namely growth conditions, that is, the existence of representations by boundary values of holomorphic functions exhibiting these conditions. The regularities marked on the abscissa are $\mathcal{P}$ for functions which are real analytic in strip-neighborhoods, $C^{\infty}$ for smooth functions, $\mathscr{D}^{\mathrm{F} \prime}$ for distributions of finite order, and $\mathcal{Q}$ for hyperfunctions.

In the lower left corner of Figure 3.1 we find the part of the Gel'fand-Shilov scheme which has been described above. The Fourier transformation is a symmetry of the diagram which operates by reflection on the diagonal, that is, exchanging growth conditions with singularity. The remarkable fact about the diagram is that it incorporates a second symmetry operating by point reflection on the center, namely duality. The combination 
of these two symmetries allowed us to draw the cross-conclusions of the above corollaries from the corresponding Paley-Wiener theorems. The self-dual $L^{2}$ in the middle is closed under Fourier transformation. It forms Gel'fand triplets together with pairs of other spaces, for example, $\left(\mathcal{P}_{-\infty}\left(\mathbb{D}^{n}\right), L^{2}\left(\mathbb{R}^{n}\right), \mathcal{Q}_{\infty}\left(\mathbb{D}^{n}\right)\right)$.

We note that similar configurations of generalized functions, which would further enhance our figure, have already been considered by Sebastião e Silva [55], Hasumi [17], and Park and Morimoto [42]. They considered the so-called Fourier ultra-hyperfunctions $\mathcal{U}$ which are the elements of the dual space of the space $\mathfrak{H}$ of entire functions of rapid decay. Via Fourier transformation, $\mathfrak{H}$ corresponds to a space $H$ of smooth functions which decay faster than $\mathrm{e}^{-\gamma|x|}$ for every $\gamma>0$, and $U$ corresponds to the space $\Lambda^{\infty}$ of distributions of exponential growth.

Note. Our definition of tempered hyperfunctions $\mathcal{Q}_{\infty}\left(\mathbb{D}^{n}\right)$ contains an inherent ambiguity: one has to make the choice whether the boundary value $F(x+i \Gamma 0) \in \mathcal{Q}_{\infty}\left(\mathbb{D}^{n}\right)$ will have fixed-growth order, say $O\left(x^{N}\right)$, as $y \in \Gamma$ tends to zero or if this growth order may vary. With our definitions, the latter is the case, for it is immediate from Definition 2.3 that $F(x+i y)$ is of a fixed-growth order in $x$ only locally uniformly in $y \in W, W$ an infinitesimal wedge of type $\Gamma$. This behavior conforms with that of boundary value representations of tempered distributions in $\varsigma^{\prime}$. As one would expect, we find the following.

Remark 3.9. There is a continuous embedding $\mathcal{P}_{-\infty}\left(\mathbb{D}^{n}\right) \hookrightarrow \diamond\left(\mathbb{R}^{n}\right)$ of test function spaces, as a consequence of Cauchy's estimates.

The above mentioned ambiguity also appears in the case of exponentially decreasing hyperfunctions (cf. [26, Note 8.3, page 411]), which explains the parentheses around $\mathcal{Q}_{*}$ and $\mathcal{P}$ in Figure 3.1: in duality to the space of infraexponential analytic functions $\mathcal{P}$ is the relative cohomology group $\mathrm{H}_{\mathbb{D}^{n}}^{n}\left(\mathbb{Q}^{n} ; \mathbb{O}_{*}\right)$ which consist of boundary values with an exponential decay at infinity that may vary with $\operatorname{Im}(z)$. This was the original definition of the space $\mathcal{Q}_{*}\left(\mathbb{D}^{n}\right)$ of exponentially decreasing hyperfunctions. In contrast, to obtain the Fourier transform of $\mathcal{P}$, Kaneko defined in [60] exponentially decreasing hyperfunctions as $\mathcal{Q}_{*}\left(\mathbb{D}^{n}\right)=\bigcup_{\varepsilon>0} \mathrm{e}^{-\varepsilon \sqrt{1+x^{2}}} \mathcal{Q}\left(\mathbb{D}^{n}\right)$, which consist of boundary values of constant exponential decay in $\operatorname{Im}(z)$. Note also that this ambiguity does not appear for asymptotic hyperfunctions, since obviously their defining functions remain asymptotic when approaching the real axis.

There is a natural relation between tempered hyperfunctions and tempered distributions: the embedding of the corresponding test function spaces $\mathcal{P}_{-\infty}\left(\mathbb{D}^{n}\right) \hookrightarrow \&\left(\mathbb{R}^{n}\right)$ remarked above has dense image [61, Theorem 15.5], and thus by duality yields the following result.

Remark 3.10. The space $\mathcal{\ell}^{\prime}\left(\mathbb{R}^{n}\right)$ is continuously embedded into $\mathcal{Q}_{\infty}\left(\mathbb{D}^{n}\right)$.

The question comes up naturally: which space of distributions is in the equivalent relation to asymptotic hyperfunctions? The distributions $\mathcal{K}^{\prime}$ were introduced in [16] and used by Estrada, Kanwal, and others (see $[11,12])$ for distributional asymptotic expansions. It will turn out that they are related to asymptotic hyperfunctions of modified type. We define $\mathcal{K}^{\prime}$ and recall some of its properties from $[11,13]$. 
Definition and Remark 3.11. Denote for $\gamma \in \mathbb{R}$ by $\mathcal{K}_{\gamma}(\mathbb{R})$ the space of all $\phi \in C^{\infty}(\mathbb{R})$, for which $\phi^{(k)}=O\left(|x|^{\gamma-k}\right)$ holds for $|x| \rightarrow \infty$ and every $k \in \mathbb{N}$. $\mathcal{K}_{\gamma}$ becomes a locally convex space equipped with the system

$$
\|\phi\|_{K, k, \gamma} \stackrel{\text { def }}{=} \sup _{x \in K}\left|(1+x)^{k-\gamma} \phi^{(k)}(x)\right|
$$

of seminorms for $K \subset \mathbb{R}$ compact. Then $\mathcal{K}_{\gamma}(\mathbb{R}) \hookrightarrow \mathcal{K}_{\gamma^{\prime}}(\mathbb{R})$ for $\gamma \leq \gamma^{\prime}$, and $\mathcal{K}(\mathbb{R}) \stackrel{\text { def }}{=}$ $\lim _{y \rightarrow \infty} \mathcal{K}_{\gamma}(\mathbb{R})$. The function algebra $\mathcal{K}$ is normal, that is, $\&$ is dense in $\mathcal{K}$, and $\mathcal{K}(\mathbb{R})$ is

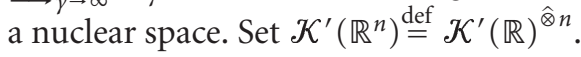

To this point, we have treated normal and modified Fourier hyperfunctions in a unified manner as far as regards notation. From now, we use the shorthand $\widetilde{\mathcal{Q}}_{-\infty}\left(\mathbb{D}^{n}\right) \stackrel{\text { def }}{=}$ $\mathcal{Q}_{-\infty}\left(\mathbb{D}^{(0,0, n)}\right)$ and $\widetilde{P}_{\infty}\left(\mathbb{D}^{n}\right) \stackrel{\text { def }}{=} \mathcal{P}_{\infty}\left(\mathbb{D}^{(0,0, n)}\right)$ for the modified type.

Proposition 3.12. There is a continuous embedding $\widetilde{P}_{\infty}\left(\mathbb{D}^{n}\right) \hookrightarrow \mathcal{K}\left(\mathbb{R}^{n}\right)$ which induces the embedding $\mathcal{K}^{\prime}\left(\mathbb{R}^{n}\right) \hookrightarrow \widetilde{\mathcal{Q}}_{-\infty}\left(\mathbb{D}^{n}\right)$.

Proof. We need only consider the case $n=1$ due to the tensor product decomposition property for $\widetilde{\mathcal{Q}}_{-\infty}\left(\mathbb{D}^{n}\right)$, see Proposition 2.5 , and the corresponding property of $\mathcal{K}^{\prime}\left(\mathbb{R}^{n}\right)$. If $f \in \widetilde{P}_{\infty}(\mathbb{D})$, then $f \in \overline{\mathcal{O}}_{m}^{B}(\overline{\bar{V}})$ for some $m \in \mathbb{N}$ and a neighborhood $V \subset \mathbb{Q}^{(0,0,1)}$ of $\mathbb{D}^{(0,0,1)}$. Without loss of generality, we can assume that $V_{\mathbb{C}}=\{z \in \mathbb{C}|| \operatorname{Im} z \mid<$ $\mathcal{E}(1+|\operatorname{Re} z|)\}$. Under this condition, it follows from elementary properties of holomorphic functions on wedge-shaped domains, see [41, Chapter 1, Theorem 4.2], that $f^{(k)} \in$ $\overline{\mathrm{O}}_{m-k}^{B}(\overline{\bar{U}})$ for every neighborhood $U$ of $\mathbb{D}^{(0,0,1)}$ which is relatively compact in $V$. This shows $f \in \mathcal{K}_{\gamma}(\mathbb{D})$ for every $\gamma>m$ and thus $\widetilde{P}_{\infty}\left(\mathbb{D}^{n}\right) \subset \mathcal{K}(\mathbb{R})$. The inclusion is continuous, since the topology of $\mathcal{K}(\mathbb{R})$ is weaker than that of $\widetilde{P}_{\infty}(\mathbb{D})$, which in turn is a consequence of Cauchy's estimates. It is also finer by definition, and the inclusion has dense image.

The counterexample $z^{m} \mathrm{e}^{i z}$ shows that the conclusion of the proposition does not hold for asymptotic hyperfunctions of ordinary type.

To conclude this section, we prove a structural theorem, as it is traditionally called in the theory of generalized functions, for the asymptotic and tempered hyperfunctions, that is, every asymptotic (tempered) hyperfunction can be rendered by applying a certain differential operator to a continuous function with the same asymptotic decay (tempered growth). Of course, a generic hyperfunction cannot be yielded through a differential operator of finite order. The right notion in this case is that of a local (pseudo)differential operator. By this we mean an infinite-order differential operator $J(D)$ with constant coefficients

$$
J(D)=\sum_{\alpha_{i} \geq 0} b_{\alpha} D_{x}^{\alpha} \quad \text { with } D_{x}^{\alpha} \stackrel{\text { def }}{=} \frac{\partial^{\alpha_{1}}}{\partial x_{1}^{\alpha_{1}}} \cdots \frac{\partial^{\alpha_{n}}}{\partial x_{n}^{\alpha_{n}}},
$$

such that the coefficients satisfy the estimate

$$
\varlimsup_{|\alpha| \rightarrow \infty} \sqrt[|\alpha|]{\left|b_{\alpha}\right| \alpha !}=0 .
$$


Those operators get their name from their property of preserving the supports of hyperfunctions, thus inducing endomorphisms of the sheaf $\mathcal{Q}$, and as well as those of $\mathcal{Q}_{ \pm \infty}$. More precisely we have by combining Theorems 2.10, 3.7, and Corollary 3.5 with [26, Proposition 8.4.8] the following.

Corollary 3.13. Every local operator $J(D)$ with constant coefficients induces an endomorphism of sheafs $\mathcal{Q}_{ \pm \infty}\left(\mathbb{R}^{n}\right) \longrightarrow \mathcal{Q}_{ \pm \infty}\left(\mathbb{R}^{n}\right)$, that is, J preserves supports, and the formula

$$
\mathscr{F}(J(D) f)(\xi)=J(\xi) \cdot(\mathscr{F} f)(\xi)
$$

holds for $f \in \mathcal{Q}_{ \pm \infty}\left(\mathbb{R}^{n}\right)$, where in particular $J(\zeta)$ is an infraexponential, entire function.

Theorem 3.14. Every $f \in \mathcal{Q}_{ \pm \infty}\left(\mathbb{R}^{n}\right)$ can be represented as $J(D) f_{0}(x)$, where $J(D)$ is a local differential operator and $f_{0} \in C\left(\mathbb{R}^{n}\right)$ is of asymptotic decay, that is, $f(x)=O\left(|x|^{-\infty}\right)$ if $f \in$ $\mathcal{Q}_{-\infty}\left(\mathbb{R}^{n}\right)$ and $f_{0}$ is of tempered growth, that is, $f(x)=O\left(|x|^{r}\right)$, for some $r \in \mathbb{R}$, if $f \in$ $\mathcal{Q}_{\infty}\left(\mathbb{R}^{n}\right)$.

The proof goes coarsely as follows: multiply the Fourier transform of the given hyperfunction with an entire function which is then taken to be the reciprocal of the Fourier transform of a local operator, which in turn is nothing but a multiplication operator with an entire function. This function can be chosen to decay fast enough to ensure that its inverse Fourier transform is continuous on the real axis. To ensure the existence of these multipliers, we need two basic lemmata.

Lemma 3.15 [26, Proposition 8.1.6]. Let $\phi(t)$ be a positive, monotonously increasing function on the half-axis $t \geq 1$ with $\phi(t)>1$ and $\lim _{t \rightarrow \infty} \phi(t)=\infty$. Then the infinite product

$$
J(\zeta)=\prod_{k=1}^{\infty}\left(1+\frac{\zeta^{2}}{(k \phi(k))^{2}}\right)
$$

with $\zeta^{2} \stackrel{\text { def }}{=} \zeta_{1}^{2}+\cdots+\zeta_{n}^{2}$, is an infraexponential, entire function which fulfills the lower estimate

$$
|J(\zeta)| \geq C \exp \left(c \frac{|\zeta|}{\phi(|\zeta|+1)}\right)
$$

for $|\operatorname{Im} \zeta| \leq \max \{(1 / \sqrt{3})|\operatorname{Re} \zeta|, 1\}$ and constants $C, c>0$.

LEMma 3.16 [26, Lemma 8.1.7]. Let $\left\{f_{k}(t)\right\}_{k \in \mathbb{N}}$ be a sequence of positive, continuous functions on the half-axis $t \geq 0$ that have infraexponential growth. Then there exists a function $\phi(t)$ as in the assumption of Lemma 3.15, and constants $C_{k}$ such that

$$
f_{k}(t) \leq C_{k} \exp \left(\frac{t}{\phi(t+1)}\right)
$$

holds for $t \geq 0$. 
Proof of Theorem 3.14. We first consider the case $f \in \mathcal{Q}_{-\infty}\left(\mathbb{R}^{n}\right)$. By decomposing $f$ linearly into components decreasing exponentially outside chosen cones, we can assume that the Fourier transform of $f$ can be represented as a single boundary value $\hat{f}(\xi)=\hat{F}(\xi+$ $i \Gamma 0)$, and such that the holomorphic function $\hat{F} \in \mathcal{O}^{*}(W)$ on an infinitesimal wedge $W$ of type $\mathbb{D}^{n}+i \Gamma$ is exponentially decreasing outside another closed cone $\Delta^{\circ}$. Let $\left\{K_{j}\right\}_{j \in \mathbb{N}}$ be an exhausting sequence of compact sets in $\mathbb{Q}^{n}$ for $W$. Then, every one of the functions $h_{j}=\sup _{\eta \in K_{j}}|\hat{F}(\xi+i \eta)|$ is infraexponential and we can apply the two cited lemmata to conclude that there exists a positive, infraexponential, entire function $J(\zeta)$ and constants $\left\{C_{j}\right\}_{j \in \mathbb{N}}$ such that $\left|\hat{F}_{j}(\xi+i \eta)\right| \leq C_{j}|J(\xi+i \eta)|$ holds for $\eta \in K_{j}$. By Theorem 3.7, $\hat{F}(\xi)$ is a $C^{\infty}$-function on the real axis. Consider the function

$$
\widehat{F}_{0}(\zeta)=\frac{\hat{F}(\zeta)}{J(\zeta)\left(1+\zeta^{2}\right)^{n}}
$$

which is for $\operatorname{Im} \zeta=\eta$ and $\eta \in W$ absolutely integrable in $\xi=\operatorname{Re} \zeta$ and its restriction to the real axis is $C^{\infty}$. Thus the inverse Fourier transform of $\hat{F}_{0}$ are well-defined in the sense of Fourier hyperfunctions as a boundary value $F_{0}=\mathscr{F}^{-1} \hat{F}_{0}$ on an infinitesimal wedge of type $\mathbb{D}^{n}+i \Delta 0$ which is furthermore exponentially decreasing outside $-\Gamma_{j}^{\circ}$. Now, $F_{0}$ can be extended to a continuous function on the real axis, which can be shown to be of asymptotic decay, since it is the inverse Fourier transform of a smooth function. Set $f_{0}(x)=F_{0}(x)$, then Corollary 3.13 shows the claim. The case $f \in \mathcal{Q}_{\infty}\left(\mathbb{R}^{n}\right)$ follows by similar reasoning, except for the following modification: the Fourier transform $\mathscr{F} f$ becomes a distribution of finite order in this case and one can apply the well-known structural theorem for these, see, for example, [61], to represent it as a finite-order differential operator applied to a continuous function. After dividing by $J(\zeta)\left(1+\zeta^{2}\right)^{n}$ and inverse Fourier transformation, one can conclude that $f_{0}$ becomes a continuous function with a certain polynomial, that is, tempered growth.

\section{Asymptotic expansions}

4.1. One-dimensional asymptotic expansions. We are now ready to generalize the moment asymptotic expansions of distributions $[12,13]$ to the case of asymptotic hyperfunctions. We start with the one-dimensional case.

Definition 4.1. For any asymptotic hyperfunction $f$ on $\mathbb{D}$ and every number $n=0,1, \ldots$, its $n$th moment is defined by

$$
\mu^{n}(f) \stackrel{\text { def }}{=} \int_{\mathbb{D}} x^{n} \cdot f(x) \mathrm{d} x
$$

(and write $\mu^{n}$ for short if there is no danger of confusion). The space

$$
\mathcal{Q}_{[N]}(\mathbb{D}) \stackrel{\text { def }}{=}\left\{f \in \mathcal{Q}_{-\infty}(\mathbb{D}) \mid \mu^{n}(f)=0, n=0, \ldots, N-1\right\}
$$

is called the remainder space of order $N$. 
Here, the integral over an asymptotic hyperfunction is defined as usual by the integral over a defining function. It is now an easy task to show the validity of asymptotic expansions for hyperfunctions on $\mathbb{D}$.

THeOREM 4.2. The moment asymptotic expansion

$$
f(x)=S_{f}^{N}(x) \bmod \mathcal{Q}_{[N+1]}(\mathbb{D}),
$$

with the asymptotic sum of order $N$ given by

$$
S_{f}^{N}(x) \stackrel{\text { def }}{=} \sum_{n=0}^{N} \frac{(-1)^{n}}{n !} \mu^{n}(f) \delta^{(n)}(x),
$$

holds for any $f \in \mathbb{Q}_{-\infty}(\mathbb{D})$.

Proof. We have to show that the remainder of the Nth-order expansion is in the remainder space of the same order:

$$
R_{f}^{N}(x) \stackrel{\text { def }}{=} f(x)-S_{f}^{N}(x) \in \mathcal{Q}_{[N+1]}(\mathbb{D})
$$

for every $N \in \mathbb{N}$. The partial sum $S_{f}^{N}$ is trivially a hyperfunction with support in the origin. Since the exponentially decaying Cauchy-Hilbert kernel $h_{z}$ of the last section induces a continuous embedding of $\mathcal{B}_{*}(\mathbb{R})$ into $\mathcal{Q}_{-\infty}(\mathbb{D})$, we can regard $S_{f}^{N}$ as an asymptotic hyperfunction. Thus the difference $R_{f}^{N}=f-S_{f}^{N}$ is again asymptotic and $R_{f}^{N} \in \mathcal{Q}_{[N+1]}(\mathbb{D})$ follows immediately from the definition of the remainder.

In applications of asymptotic expansions, as well as for the expansions of distributions as in $[11,12,13]$, it is useful to state the expansion in the so-called parametric form, that is, in application to a test function with scaled argument. We start with a technical lemma.

Lemma 4.3. For any continuous seminorm $\|\cdot\|$ on $\mathcal{P}_{\infty}(\mathbb{R})$ and every function $\phi \in \mathcal{P}_{\infty}(\mathbb{R})$ with $\phi^{(n)}(0)=0$ for $n=0, \ldots, N$ holds

$$
\left\|\phi\left(\frac{z}{\lambda}\right)\right\|=O\left(|\lambda|^{-N-1}\right)
$$

for $|\lambda| \rightarrow \infty$.

Proof. Similar to the proof of Theorem 3.4, we represent $\mathcal{P}_{\infty}(\mathbb{D})$ as an inductive limit $\mathcal{P}_{\infty}(\mathbb{D}) \cong \lim _{\overline{0}}^{B}\left(\overline{\overline{U_{m}}}\right), m \in \mathbb{N}$, with neighborhoods $U_{m}=\{z \in \mathbb{C}|| \operatorname{Im} z \mid<1 / m\}$. A locally convex topology on $\overline{\mathrm{O}}_{m}^{B}\left(\overline{\bar{U}_{m}}\right)$ can be generated by the system of seminorms

$$
\|\phi(z)\|_{m, R} \stackrel{\text { def }}{=} \sup \left\{\left|\phi(z)\left(1+|\operatorname{Re} z|^{-m}\right)\right|\left|z \in \overline{U_{m}},\right| \operatorname{Re} z \mid \leq R\right\}
$$

for $R>0$. This topology is apparently weaker than the original topology of the space $\overline{\mathrm{O}}_{m}^{B}\left(\overline{\overline{U_{m}}}\right)$, but has the following property, which is sufficient for our purpose: if $\phi_{n}$ is a sequence which is bounded in $\overline{\mathrm{O}}_{m}^{B}\left(\overline{\overline{U_{m}}}\right)$ and converges to zero in the seminorms $\|\cdot\|_{m, R}$ for every $R>0$, then it converges to zero in $\overline{0}_{m^{\prime}}^{B}\left(\overline{\overline{U_{m^{\prime}}}}\right)$ for every $m^{\prime}>m$ (cf. Definition 2.3 ). 
That means, the systems of seminorms $\|\cdot\|_{m, R}$ induces on the bounded subsets of $\mathcal{P}_{\infty}(\mathbb{D})$ the original topology of that space. Now, for any $\phi \in \mathcal{P}_{\infty}(\mathbb{D})$ the set $\{\phi(z / \lambda)\}_{\lambda>C>0}$ is bounded in $\mathcal{P}_{\infty}(\mathbb{D})$ and therefore it is also bounded in some ${\overline{0_{m}}}_{m}^{B}\left(\overline{\overline{U_{m}}}\right)$. We can thus argue as follows: let $\phi \in \overline{\mathrm{O}}_{m}^{B}\left(\overline{\overline{U_{m}}}\right)$ such that $\phi^{(n)}(0)=0$ for $n=0, \ldots, N$. Then, there is a constant $K>0$ for which

$$
|\phi(z)| \leq K|\operatorname{Re} z|^{N+1}, \quad z \in U_{m},|\operatorname{Re} z| \leq 1 .
$$

If $\lambda>R$, then $\|\phi(z / \lambda)\|_{m, R} \leq K / \lambda^{N+1}$, and thus

$$
\left\|\phi\left(\frac{z}{\lambda}\right)\right\|_{m, R}=O\left(\left|\lambda^{-N-1}\right|\right)
$$

for all $R$. Since the topology of $\mathcal{P}_{\infty}(\mathbb{R})$ is generated by all seminorms for which the restriction to every subspace ${\overline{0_{m}}}_{m}^{B}\left(\overline{\overline{U_{m}}}\right)$ is again a continuous seminorm, the assertion follows.

A lemma of this type is fundamental for every asymptotic expansion in parametric form, as the following general arguments will show.

THEOREM 4.4. For every $f \in \mathbb{Q}_{-\infty}(\mathbb{D})$, the moment asymptotic expansion in parametric form

$$
f(\lambda x) \sim \sum_{n=0}^{\infty} \frac{(-1)^{n} \mu^{n}(f) \delta^{(n)}(x)}{n ! \lambda^{n+1}}
$$

holds for $\lambda \in \mathbb{R},|\lambda| \rightarrow \infty$. This formula holds in the dual sense, that is, for all $\phi \in \mathcal{P}_{\infty}(\mathbb{D})$ holds

$$
\langle f(\lambda x), \phi(x)\rangle=\sum_{n=0}^{N} \frac{\mu^{n}(f) \phi^{(n)}(0)}{n ! \lambda^{n+1}}+O\left(|\lambda|^{-N-2}\right)
$$

for $\lambda \in \mathbb{R},|\lambda| \rightarrow \infty$.

Proof. If $f$ and $\phi$ are as above, then

$$
\langle f(\lambda x), \phi(x)\rangle=\frac{1}{\lambda}\left\langle f(x), \phi\left(\lambda^{-1} x\right)\right\rangle .
$$

We write the Taylor expansion of $\phi(x / \lambda)$ in $x$ around 0 with remainder as

$$
\phi\left(\frac{x}{\lambda}\right)=\sum_{n=0}^{N} \frac{\phi^{(n)}(0)}{n ! \lambda^{n}} \cdot x^{n}+T_{N}(\lambda ; x) .
$$

Since $R_{f}^{N} \in \mathcal{Q}_{[N+1]}(\mathbb{D})$, it follows that

$$
\begin{aligned}
\langle f(\lambda x), \phi(x)\rangle & =\left\langle S_{f}^{N}(\lambda x)+R_{f}^{N}(\lambda x), \phi(x)\right\rangle \\
& =\sum_{n=0}^{N} \frac{\mu^{n}(f) \phi^{(n)}(0)}{n ! \lambda^{n+1}}+\lambda^{-1}\left\langle R_{f}^{N}(x), T_{N}(\lambda ; x)\right\rangle .
\end{aligned}
$$


Now, $T_{N}(\lambda ; x)$ is in $\mathcal{P}_{\infty}(\mathbb{D})$ and $D_{x}^{n} T_{N}(\lambda ; 0)=0, n=0, \ldots, N$. Consequently, the assertion follows from the above lemma, the definition of $T_{N}$, and the fact that $R_{f}^{N}$ is a continuous linear functional on $\mathcal{P}_{\infty}(\mathbb{D})$.

We state clearly at this point that, despite the synonymy, our asymptotic expansions of hyperfunctions have almost nothing to do with that based on second microlocalization, see [27]. Rather it is a proper generalization of the distributional expansions by Estrada and Kanwal [12, 13].

It is simple but useful to restate the expansion for the Fourier transforms of asymptotic hyperfunctions.

Proposition 4.5. Let $f \in \mathcal{Q}_{-\infty}(\mathbb{D})$. Then, its Fourier transform $\hat{f}$ fulfills the asymptotic expansion

$$
\widehat{f}(\xi)=\sum_{n=0}^{N} \frac{(-i \xi)^{n}}{n !} \mu^{n}(f) \bmod \widehat{\mathcal{Q}}_{[N+1]}(\mathbb{R})
$$

around 0 , that is, for $|x| \rightarrow 0$. Here, the image of $\mathcal{Q}_{[N+1]}(\mathbb{D})$ under the Fourier transformation is denoted by

$$
\widehat{\mathcal{Q}}_{[N+1]}(\mathbb{R}) \stackrel{\text { def }}{=}\left\{g \in C^{\infty *}(\mathbb{R}) \mid g^{(n)}(0)=0, n=0, \ldots, N\right\} .
$$

Proof. By Theorem 3.7, we can calculate the derivatives of $\hat{f}$ at 0 explicitly:

$$
i^{k} \hat{f}^{(k)}(0)=\left.\left(i^{k} \frac{\mathrm{d}^{k}}{\mathrm{~d} \xi^{k}} \int_{\mathbb{D}} \mathrm{e}^{-i x \xi} f(x) \mathrm{d} x\right)\right|_{\xi=0}=i^{k} \int_{\mathbb{D}}(-i x)^{k} f(x) \mathrm{d} x=\mu^{k}(f) .
$$

That is, the stated asymptotic expansion is identical to the Taylor expansion of the smooth function $\hat{f}$ around 0 and we have $\hat{R}_{N}^{(n)}(0)=0$ for $n \leq N$ by definition of the remainder space $\mathcal{Q}_{[N+1]}(\mathbb{D})$.

This allows us to derive the following basic result (cf. [12, 13]).

Proposition 4.6. The moment asymptotic expansion of asymptotic hyperfunctions is a complete asymptotic scheme, that is, for any given sequence $\left\{\mu^{n}\right\}_{n \in \mathbb{N}_{0}} \subset \mathbb{C}$, there exists an $f \in \mathbb{Q}_{-\infty}(\mathbb{D})$ with $\mu^{n}(f)=\mu^{n}$.

In order to prove this assertion, we use the classical theorem of Ritt in a formulation which can be derived from [10, Section 1].

Theorem 4.7. For $\left\{\mu^{n}\right\}_{n \in \mathbb{N}_{0}} \subset \mathbb{C}$ and a given sector $S=\{\zeta \in \mathbb{C}|\alpha<\arg \zeta<\beta, 0<| \zeta \mid\}$ with vertex 0 in the complex plane, there exists a function $\psi(\zeta)$, which is holomorphic in $S$, bounded in $\bar{S}$, satisfies $\psi(\zeta)=O\left(|\zeta|^{-\infty}\right)$ for $\zeta \rightarrow \infty$ in $S$, and furthermore such that

$$
\psi(\zeta) \sim \sum_{n=0}^{\infty} \frac{(-i \zeta)^{n}}{n !} \mu^{n}
$$

holds for $\zeta \rightarrow 0$ in $S$. 
Proof of Proposition 4.6. By Proposition 4.5 and Theorem 3.7, the assertion is equivalent to the following: there exists a function $\hat{f} \in C^{\infty *}(\mathbb{R})$ such that $\hat{f}^{(n)}(0)=(-i)^{n} \mu^{n}$, since then $f=\mathscr{F}^{-1} \hat{f} \in \mathcal{Q}_{-\infty}(\mathbb{D})$ fulfills the original condition. Choose the sector $S$ in Theorem 4.7 large enough to contain $\mathbb{R} \backslash\{0\}$ and a function $\psi$ as there. This function is analytic and therefore $C^{\infty}$ in $\mathbb{R} \backslash\{0\}$ and has a smooth continuation to the point 0 . Furthermore, it is asymptotic along the real axis, and thus we can choose $\hat{f}=\psi \in$ $C^{\infty *}(\mathbb{R})$.

We remark that $f$ can even be chosen to lie in $\&(\mathbb{R})$.

4.2. Radon transformation of asymptotic hyperfunctions. Our final task is to carry over the one-dimensional asymptotic expansions to higher dimensions. To this end, we use the geometrical Radon transformation of hyperfunctions, introduced by Takiguchi and Kaneko, see [60]. This transformation decomposes a given asymptotic hyperfunction into a one-dimensional part depending only on the radial coordinate and a second part depending on the remaining angle coordinates. For the readers convenience, we briefly review the general concept. Note that the following results, up to Definition and Theorem 4.8 are proved in [60].

For any $f \in \delta\left(\mathbb{R}^{n}\right)$, its Radon transform is defined by integrals over the affine planes $P(\omega, t) \stackrel{\text { def }}{=}\left\{x \in \mathbb{R}^{n} \mid \omega x=t\right\}$ for $\omega \in S^{n-1}$ and $t \in \mathbb{R}$ by

$$
\mathscr{R} f(\omega, t) \stackrel{\text { def }}{=} \int_{\mathbb{R}^{n}} \delta(t-\omega x) f(x) \mathrm{d} x .
$$

$\mathscr{R} f$ is a $C^{\infty}$-function on $S^{n-1}$ with values in $\&(\mathbb{R})$, that is, an element of the topological vector space $C^{\infty}\left(S^{n-1} ; \delta(\mathbb{R})\right)$ in the notation of [61, Chapters 40-44]. Since $8(\mathbb{R})$ is nuclear, this space is isomorphic to a completed tensor product and thus $\mathscr{R} f \in C^{\infty}\left(S^{n-1}\right) \hat{\otimes}$ $\wp(\mathbb{R})$ holds. It is geometrically clear that $\mathscr{R} f$ is an even function in $(\omega, t)$, that is, $\mathscr{R} f(-\omega,-t)=\mathscr{R} f(\omega, t)$.

For a hyperfunction $f$, neither its restriction to $P(\omega, t)$ nor its integral over this hyperplane is well-defined in general, see [26, Chapter 3, Section 4]. To remedy that problem, Takiguchi and Kaneko introduce a special class of Radon hyperfunctions, for which they define the Radon transformation via duality. These Radon hyperfunctions are not a proper subclass of Fourier hyperfunctions, but for appropriate subclasses of Radon hyperfunctions which are also Fourier hyperfunctions, the Radon transformation can be defined explicitly using the boundary value representation of hyperfunctions. This is especially the case for asymptotic hyperfunctions, as we will now see.

Using the decomposition

$$
\delta(t-\omega x)=\frac{-1}{2 \pi i}\left(\frac{1}{(t+i 0)-\omega x}-\frac{1}{(t-i 0)-\omega x}\right)
$$

of the $\delta$-function, we are led to the formal definition of the Radon transform for an $f \in \mathbb{Q}_{-\infty}\left(\mathbb{D}^{n}\right):$

$$
\mathscr{R} f(\omega, t) \stackrel{\text { def }}{=} G(\omega, t+i 0)-G(\omega, t-i 0)
$$


with

$$
G(\omega, \tau) \stackrel{\text { def }}{=} \frac{-1}{2 \pi i} \int_{\mathbb{D}^{n}} \frac{f(x)}{\tau-\omega x} \mathrm{~d} x
$$

for $\tau \in \mathbb{C} \backslash \mathbb{R}$. This result is to be understood as a Fourier hyperfunction-valued ordinary function in $\omega$. In a special boundary value representation $f(x)=\sum_{j=1}^{N} F_{j}\left(x+i \Gamma_{j} 0\right)$, with $F_{j} \in \mathbb{O}_{-\infty}\left(\mathbb{D}^{n}+i \Gamma_{j} 0\right)$, this becomes

$$
\mathscr{R} f(\omega, t) \stackrel{\text { def }}{=} \sum_{j=1}^{N} G_{j}(\omega, t+i 0)-G_{j}(\omega, t-i 0)
$$

with defining functions $G_{j}$ given by

$$
G_{j}\left(\omega, \tau_{j}\right) \stackrel{\text { def }}{=} \frac{-1}{2 \pi i} \int_{\operatorname{Im} z_{j}=y_{j}} \frac{F_{j}\left(z_{j}\right)}{\tau_{j}-\omega z_{j}} \mathrm{~d} z_{j}=\frac{-1}{2 \pi i} \int_{\mathbb{R}^{n}} \frac{F_{j}\left(x+i y_{j}\right)}{t+i s_{j}-\omega\left(x+i y_{j}\right)} \mathrm{d} x .
$$

Here, we set $\tau_{j}=t+i s_{j}$ and $z_{j}=x+i y_{j}$ for $y_{j} \in \Gamma_{j}$. The integrals converge absolutely and independently of the damping factor $\left(\tau_{j}-\omega z_{j}\right)^{-1}$, and yield a defining function for $\mathscr{R} f$. Furthermore, the definition is independent of the choice of integration planes $\operatorname{Im} z_{j}=y_{j}$ by Cauchy's theorem. In the sense that for $\omega \rightarrow \omega_{0} \in S^{n-1}$ one has $G_{j}\left(\omega, \tau_{j}\right) \rightarrow G_{j}\left(\omega_{0}, \tau_{j}\right)$ in the topology of $\mathbb{O}^{*}(\mathbb{Q} \backslash \mathbb{D})$, we can regard $\mathscr{R} f(\omega, t)$ as a continuous function on $S^{n-1}$. That is, we have $\mathscr{R} f \in C\left(S^{n-1} ; \mathcal{Q}\left(\mathbb{D}_{t}\right)\right) \cong C\left(S^{n-1}\right) \hat{\otimes} \mathcal{Q}\left(\mathbb{D}_{t}\right)$, where $\mathbb{D}_{t}$ denotes $\mathbb{D}$ with coordinate $t$. We will see below that the Radon transformation is well-defined, that is, does not depend on the choice of boundary value representation of $f$, by exhibiting its connection with the Fourier transformation.

To see that $\mathscr{R} f$ is an asymptotic hyperfunction in $t$ for all $\omega$, we first recover the original representation of the Radon transform by integrals over hyperplanes from the above definition of the $G_{j}$ :

$$
G_{j}\left(\omega, \tau_{j}\right)=\int_{\mathbb{R}^{n}} \delta\left(t+i s_{j}-\omega\left(x+i y_{j}\right)\right) F_{j}\left(x+i y_{j}\right) \mathrm{d} x=\int_{P\left(\omega, \tau_{j}\right)} F_{j}\left(x+i y_{j}\right) \mathrm{d} S .
$$

Here, $P\left(\omega, \tau_{j}\right)$ is the $n-1$-dimensional real affine plane

$$
P\left(\omega, \tau_{j}\right) \stackrel{\text { def }}{=}\left\{z_{j}=x+i y_{j} \mid t+i s_{j}=\omega\left(x+i y_{j}\right)\right\} \subset \mathbb{C}^{n} .
$$

Now, the estimate $|x|=O(|t|)$ holds locally uniformly in $s_{j}$ and $y_{j}$ on $P\left(\omega, \tau_{j}\right)$. From this it follows that $G_{j}$ is an asymptotic function in $t$ locally uniformly in $y_{j}$. Thus indeed, $\mathscr{R} f(\omega, \cdot) \in \mathcal{Q}_{-\infty}\left(\mathbb{D}_{t}\right)$ for all $\omega \in S^{n-1}$.

There is a close connection between the Radon and the Fourier transformations, which is given by the formula

$$
\mathscr{R} f(\omega, t)=\frac{1}{2 \pi} \int_{-\infty}^{\infty} \hat{f}(\rho \omega) \mathrm{e}^{i t \rho} \mathrm{d} \rho
$$


For $f(x) \in \mathcal{Q}_{-\infty}\left(\mathbb{D}^{n}\right)$ we have $\hat{f}(\xi) \in C^{\infty *}\left(\mathbb{R}^{n}\right)$ by Theorem 3.7, and its partial back transformation along the fiber $\xi=\rho \omega$ can be interpreted as a Fourier hyperfunction as follows: split the integration path at 0 and compute the two components

$$
G_{ \pm}(\omega, \tau) \stackrel{\text { def }}{=} \pm \frac{1}{2 \pi} \int_{0}^{ \pm \infty} \hat{f}(\rho \omega) \mathrm{e}^{i \tau \rho} \mathrm{d} \rho
$$

of the defining function of $\mathscr{R} f$ for $\pm \operatorname{Im} \tau>0$ separately. We show that this yields the same result as the original formula for $G(\omega, \tau)$ :

$$
G_{ \pm}(\omega, \tau)=\frac{1}{2 \pi} \int_{0}^{ \pm \infty} \mathrm{e}^{i \tau \rho}\left(\int_{\mathbb{D}^{n}} f(x) \mathrm{e}^{i \rho \omega x} \mathrm{~d} x\right) \mathrm{d} \rho
$$

for $\pm \operatorname{Im} \tau>0$. The inner integral is an absolutely convergent integral over defining functions and can thus be exchanged with the outer one, which leads to

$$
G_{ \pm}(\omega, \tau)=\int_{\mathbb{D}^{n}} f(x)\left(\frac{1}{2 \pi} \int_{0}^{ \pm \infty} \mathrm{e}^{i \rho(\tau-\omega x)} \mathrm{d} \rho\right) \mathrm{d} x=\int_{\mathbb{D}^{n}} \frac{-1}{2 \pi i} \frac{f(x)}{\tau-\omega x} \mathrm{~d} x=G(\omega, \tau),
$$

as we wanted to show. As an aside, this also shows the invariance of the definition of $\mathscr{R} f$ under a change of the boundary value representation of $f$ by namely the property of the Fourier transformation, see, for example, [26, Lemma 8.3.3 and Theorem 8.3.4]. We compile the information we won so far in the following.

Definition and Theorem 4.8. Define the Radon transform of $f(x) \in \mathbb{Q}_{-\infty}\left(\mathbb{D}^{n}\right)$, denoted by $\mathscr{R} f(\omega, \cdot)$, by the functions $G_{ \pm}\left(\omega, \tau_{ \pm}\right) \in C^{\infty}\left(S^{n-1} ; \mathbb{O}_{-\infty}\left(\mathbb{Q}_{ \pm}\right)\right)$, called canonical defining functions, and where $\mathbb{Q}_{ \pm} \stackrel{\text { def }}{=} \overline{\overline{\{z \mid \operatorname{Im} z \gtrless 0\}}} \backslash \mathbb{D}$, via the boundary values

$$
\mathscr{R} f(\omega, t)=[G(\omega, \tau)]_{\tau=t}=\left[G_{+}(\omega, t+i s), G_{-}(\omega, t-i s)\right]_{s=0} .
$$

It holds that $\mathscr{R} f \in C^{k}\left(S^{n-1}\right) \hat{\otimes} \mathcal{Q}_{-\infty}\left(\mathbb{D}_{t}\right), \mathscr{R} f$ is an even function in $(\omega, t)$, and the canonical defining function $G(\omega, \tau)$ fulfills the estimates

$$
\left|D_{\omega}^{\alpha} G(\omega, \tau)\right| \leq C \frac{(|\alpha| !)^{2}}{v^{|\alpha|}}
$$

with constants $v, C>0$.

Proof. We only have to show the bounds on the derivatives of $G$. The proof follows [60, Propositions 2.3 and 2.8]. As in Theorem 3.7, we can assume $f$ to be represented by a single boundary value $f=F(x+i \Gamma 0), F \in O_{-\infty}\left(\mathbb{D}^{n}+i \Gamma 0\right)$, such that $F$ decreases exponentially outside a certain cone $\Delta^{\circ}$. A coarse estimate for the Fourier transform of $F$ is

$$
\left|D_{\xi}^{\alpha} \hat{F}(\zeta)\right| \leq\left|\int_{\mathbb{R}^{n}} \mathrm{e}^{-i z \zeta}(-i z)^{\alpha} F(z) \mathrm{d} x\right| \leq C \int_{\mathbb{R}^{n}} \mathrm{e}^{x \eta+y \xi}\left|x^{\alpha}\right||F(x+i y)| \mathrm{d} x,
$$

with $\zeta=\xi+i \eta$ and $z=x+i y$. Now, for every compact set $L \subset-\Delta$ and $\eta \in L$, there is a constant $\delta_{L}>0$ such that we can further estimate

$$
\left|D_{\xi}^{\alpha} \widehat{F}(\zeta)\right| \leq C^{\prime} \mathrm{e}^{y \xi} \int_{\mathbb{R}^{n}} \mathrm{e}^{-\delta_{L}|x|}\left|x^{\alpha}\right||F(x+i y)| \mathrm{d} x \leq C^{\prime \prime} \cdot \frac{|\alpha| !}{\delta_{L}^{|\alpha|}} \cdot \mathrm{e}^{y \xi}
$$


(cf. the proof of [26, Theorem 8.3.2]). Since the derivatives $D_{\omega}^{\alpha} \hat{f}(\rho \omega)$ exist for arbitrary $\alpha$, we can calculate that of, for example, $G_{+}$:

$$
\left|D_{\omega}^{\alpha} G_{+}(\omega, t+i s)\right|=\left|\frac{1}{2 \pi} D_{\omega}^{\alpha} \int_{0}^{\infty} \mathrm{e}^{i(\rho+i \sigma)(t+i s)} \hat{F}((\rho+i \sigma) \omega) \mathrm{d} \rho\right|,
$$

where $\omega \in-\Delta$ if we choose $\sigma>0$. This can be estimated by

$$
\leq\left|\frac{1}{2 \pi} \int_{0}^{\infty} \mathrm{e}^{-\rho s-\sigma t} \rho^{|\alpha|}\left(D_{\xi}^{\alpha} \hat{F}\right)((\rho+i \sigma) \omega) \mathrm{d} \rho\right| .
$$

Using the first estimate above, we further get for every $\kappa>0$,

$$
\leq C \int_{0}^{\infty} \rho^{|\alpha|} \frac{|\alpha| !}{\delta_{\sigma}^{|\alpha|}} \mathrm{e}^{\kappa \rho} \mathrm{e}^{-\rho s-\sigma t} \mathrm{~d} \rho \leq C^{\prime} \frac{(|\alpha| !)^{2}}{\left(\delta_{\sigma}(s-\kappa)\right)^{|\alpha|}} \mathrm{e}^{-\sigma t} .
$$

Since this final result is finite for every $\sigma$, we can replace the denominator by $v^{|\alpha|}$ for a suitable constant $v>0$. This proves the assertion.

The regularity of $\mathscr{R} f$ expressed by the bounds on its derivatives in $\omega$, is the well-known one of ultradifferentiable functions (cf. [31]).

Definition 4.9. A function $g \in C^{\infty}\left(\mathbb{R}^{n}\right)$ is said to be in the Gevrey class of order $s$ for $s>1$, if and only if for every compact set $K \subset \mathbb{R}^{n}$ there are constants $h$ and $C>0$ such that

$$
\left\|D^{\alpha} g\right\|_{C^{0}(K)} \leq C h^{|\alpha|}(|\alpha| !)^{s}
$$

holds for $|\alpha|=0,1,2, \ldots$.

For $f \in \mathbb{Q}_{-\infty}\left(\mathbb{D}^{n}\right)$, we immediately find by Definition and Theorem 4.8 that Gevrey bounds hold for all derivatives of $\mathscr{R} f(\omega, \cdot)$ with respect to $\omega \in S^{n-1}$, for $h=v^{-1}$ and with $s=2$. This entails the following.

Corollary 4.10. For $f \in \mathcal{Q}_{-\infty}\left(\mathbb{D}^{n}\right), \mathscr{R} f(\omega, t)$ is a function in the Gevrey class of order 2 on $S^{n-1}$ with values in $\mathcal{Q}_{-\infty}\left(\mathbb{D}_{t}\right)$.

This result is analogous to [60, Proposition 2.8], where it is shown that this type of regularity holds for the Radon transforms of exponentially decaying hyperfunctions, and is the best possible in this case.

4.3. Radon asymptotic expansions. The moments of a Radon transform $\mathscr{R} f$, which are the last ingredients for asymptotic expansions in higher dimensions are known as the Helgason moments, see [18], and are defined by the formula

$$
p_{\Re f}^{k}(\omega) \stackrel{\text { def }}{=} \mu^{k}(\mathscr{R} f(\omega, \cdot))=\int_{\mathbb{D}_{t}} t^{k} \cdot \mathscr{R} f(\omega, t) \mathrm{d} t \quad \text { for } k=0,1,2, \ldots
$$

The $p_{\mathscr{R} f}^{k}(\omega)$ satisfy the Helgason moment condition (cf. [18, page 100]).

Proposition 4.11. For $f \in \mathcal{Q}_{-\infty}\left(\mathbb{D}^{n}\right)$ and $k=0,1,2, \ldots$, the Helgason moment $p_{\mathscr{R} f}^{k}(\omega)$ is a homogeneous polynomial of total degree $k$ in $\omega$. 
Proof. We first calculate the Helgason moments as in the proof of Proposition 4.5, to obtain

$$
\begin{aligned}
p_{\mathscr{R} f}^{k}(\omega) & =\left.\left(\int_{\mathbb{D}_{t}} \mathscr{R} f(\omega, t) t^{k} \mathrm{e}^{-i t \rho} \mathrm{d} t\right)\right|_{\rho=0} \\
& =\left.\left(i^{k} \frac{\mathrm{d}^{k}}{\mathrm{~d} \rho^{k}} \int_{\mathbb{D}_{t}} \mathscr{R} f(\omega, t) \mathrm{e}^{-i t \rho} \mathrm{d} t\right)\right|_{\rho=0}=\left.\left(i^{k} \frac{\mathrm{d}^{k}}{\mathrm{~d} \rho^{k}} \hat{f}(\rho \omega)\right)\right|_{\rho=0} .
\end{aligned}
$$

Since $\hat{f}$ is $C^{\infty}$, we can write down its Taylor series up to the order $k$ with remainder around the point 0 as

$$
\widehat{f}(\xi)=\sum_{|\alpha| \leq k} a_{\alpha} \xi^{\alpha}+R_{k+1}(\xi)
$$

with $a_{\alpha}=D_{\xi}^{\alpha} \hat{f}(0) / \alpha$ ! and

$$
R_{k+1}(\xi)=\sum_{|\beta|=k+1} \frac{D_{\xi}^{\beta} \hat{f}(\Theta \xi)}{\beta !} \xi^{\beta}
$$

for a suitable $\Theta \in[0,1]$. Inserting this in formula (4.40) above yields, since we have $D_{\rho}^{k} R_{k+1}(\rho \omega)=O(|\rho|)$ for $|\rho| \rightarrow 0$, the exact form of $p_{\mathscr{R} f}^{k}(\omega)$, namely,

$$
p_{\Re f}^{k}(\omega)=i^{k} k ! \sum_{|\alpha|=k} a_{\alpha} \omega^{\alpha}
$$

proving the assertion.

Now, we have collected all necessary facts about the Radon transformation of asymptotic hyperfunctions, their Helgason moments, and the relation to their Fourier transforms to carry over the asymptotic expansions of Theorem 4.2 and Proposition 4.5 to the case of dimension $n>1$.

Theorem 4.12. For $f \in \mathbb{Q}_{-\infty}\left(\mathbb{D}^{n}\right)$, the Radon asymptotic expansion

$$
\mathscr{R} f(\omega, t)=\sum_{k=0}^{N} \frac{(-1)^{k}}{k !} p_{\mathscr{R} f}^{k}(\omega) \cdot \delta^{(k)}(t) \bmod \mathscr{R} \mathcal{Q}_{[N+1]}\left(S^{n-1} \times \mathbb{D}_{t}\right)
$$

holds. Here, the Radon remainder space of order $N+1$ was defined by

$$
\mathscr{R} \mathcal{Q}_{[N+1]}\left(S^{n-1} \times \mathbb{D}_{t}\right) \stackrel{\text { def }}{=}\left\{\mathscr{R} g \in \mathscr{R} \mathcal{Q}_{-\infty}\left(\mathbb{D}^{n}\right) \mid p_{\mathscr{R} g}^{k} \equiv 0, k=0, \ldots, N\right\}
$$

Furthermore, the following relation to the Taylor expansion of the Fourier transform $\hat{f}$ around the origin holds:

$$
\mathscr{R} f(\omega, t)=\sum_{k=0}^{N} \sum_{|\alpha|=k}(-i)^{k} a_{\alpha} \omega^{\alpha} \cdot \delta^{(k)}(t) \bmod \mathscr{R} \mathcal{Q}_{[N+1]}\left(S^{n-1} \times \mathbb{D}_{t}\right),
$$

where $a_{\alpha}=D_{\xi}^{\alpha} \hat{f}(0) / \alpha !$ 
Proof. By [60, Theorem 2.4], the Nth partial sum $S_{\mathscr{R} f}^{N}$ of the Radon asymptotic expansion has a unique preimage under the Radon transformation. By the same theorem, this preimage $\mathscr{R}^{-1} S_{\mathscr{R} f}^{N}$ is a hyperfunction with compact support and therefore especially in $\mathcal{Q}_{-\infty}\left(\mathbb{D}^{n}\right)$. Thus, the remainder $f-\mathscr{R}^{-1} S_{\mathscr{R} f}^{N}$ is asymptotic and its image under $\mathscr{R}$ is exactly the remainder of the Radon asymptotic expansion. The Helgason moments of the remainder vanish up to order $N$ by definition.

Note aside how every partial sum $S_{\mathscr{R} f}^{N}$ becomes an even function in $(\omega, t)$ : when changing the variable to $(-\omega,-t)$, the sign of $\delta^{(k)}$ cancels that of $p_{\Re f}^{k}$. We refrain from rewriting the Radon asymptotics in parametric form as in Theorem 4.4 , and instead give a very basic example.

Example 4.13. Let $f(x)=J(D) \delta(x-a)$, with $a \in \mathbb{R}^{n}$, and let $J(D)=\sum_{|\alpha| \geq 0} b_{\alpha} D_{x}^{\alpha}$ be a local operator. The Radon transform of $f$ is

$$
\mathscr{R} f(\omega, t)=J\left(\omega D_{t}\right) \delta(t-a \omega)
$$

see [60, Example 3.3], by which the Helgason moments can easily be calculated. This yields the Radon asymptotic expansion

$$
\mathscr{R} f(\omega, t) \sim \sum_{k=0}^{\infty} \sum_{|\alpha| \leq k} \frac{(-1)^{k-|\alpha|}}{|\alpha| !} \cdot\left(b_{\alpha} \omega^{\alpha}\right)(a \omega)^{k-|\alpha|} \cdot \delta^{(k)}(t)
$$

This representation makes the Helgason moment condition manifest.

We conclude our analysis by giving a condition on the support of a hyperfunction in terms of the moments of its Radon asymptotic expansion. To do so, we apply a recent result by Kim et al.

Theorem 4.14 [66, Theorem 3.1]. A sequence $\left\{\mu^{k}\right\}_{k \in \mathbb{N}_{0}}$ is the moment sequence of a hyperfunction $f \in \mathscr{B}_{*}(\mathbb{R})$ with support in the interval $[-R, R]$, if and only if for every $S>R$ and $\varepsilon>0$ there exists a constant $C_{\varepsilon}>0$ such that the estimate

$$
\left|\sum_{k=0}^{\infty} \frac{\mu^{k}}{k !} \frac{1}{2 S}\left(-\frac{\pi i q}{S}\right)^{k}\right| \leq C_{\varepsilon} \mathrm{e}^{\varepsilon|q|}
$$

holds for all $q \in \mathbb{Z}$.

This result was originally formulated for cubic domains in $\mathbb{R}^{n}$, but for our purpose a one-dimensional version suffices, since we want to use the Radon transformation again to generalize to higher dimensions. To that end, we need an adaption of another result of [60], which connects the support of a Radon transform with that of its Radon-preimage. This so-called support theorem goes back to Helgason, see [18, page 105], where it is proved for functions in $\mathcal{D}\left(\mathbb{R}^{n}\right)$. 
THEOREM 4.15 [60, Theorem 4.1]. If $f \in \mathbb{Q}_{-\infty}\left(\mathbb{D}^{n}\right)$ is such that $\mathscr{R} f(\omega, t)$ vanishes for $|t| \geq R$, then $f(x)$ vanishes for $|x| \geq R$.

We finally give the intended result which allows one to restrict the support of hyperfunctions to spherical domains, provided that a growth condition on the Helgason moments of the Radon asymptotic expansion holds.

Theorem 4.16. A sequence $\left\{\alpha^{k}(\omega)\right\}_{k \in \mathbb{N}_{0}}$ of polynomials on $S^{n-1}$ which fulfill Helgason's moment condition is the sequence of Helgason moments of the Radon transform $\mathscr{R} f$ of a hyperfunction $f \in \mathscr{B}_{*}\left(\mathbb{R}^{n}\right)$ with support in the ball $B_{R}(0)=\left\{x \in \mathbb{R}^{n}|| x \mid \leq R\right\}$, if and only if the $\alpha^{k}$ satisfy estimate (4.49).

Proof. If $f \in \mathcal{Q}_{-\infty}\left(\mathbb{D}^{n}\right)$ has support in $B_{R}(0)$, then $\mathscr{R} f(\omega, t)$ vanishes for $|t|>R$ and all $\omega$. The estimate is then immediate from Theorem 4.14. Let conversely $\alpha^{k}(\omega)$ be a sequence of polynomials satisfying Helgason's condition and estimate (4.49). From Proposition 4.6 and the formula for the Helgason moments found in the proof of Proposition 4.11 it follows that there is an asymptotic hyperfunction $g \in \mathcal{Q}_{-\infty}\left(\mathbb{D}^{n}\right)$ such that $p_{\mathscr{R} g}^{k}(\omega)=$ $\alpha^{k}$. By the assumption and Theorem $4.14, g$ can be chosen such that every component $\mathscr{R} g(\omega, \cdot)$ has compact support in $[-R, R]$. Then $g$ itself vanishes for $|x|>R$ by Theorem 4.15 .

We finally want to give a very simple example that shows how the Radon asymptotic expansion can be utilized to solve differential equations by an asymptotic series ansatz. Naturally, the Radon transform becomes most effective when the equation in question exhibits a spherical symmetry.

Example 4.17. Consider the ordinary differential equation on $\mathbb{D}^{n}$

$$
\left(r^{2} \frac{\mathrm{d}}{\mathrm{d} r}-1\right) f(x)=0 \quad \text { where } r=\left(\sum_{i=1}^{n} x_{i}^{2}\right)^{1 / 2}
$$

After Radon transformation this is easily seen to correspond to the equation

$$
\left(t^{2} \frac{\mathrm{d}}{\mathrm{d} t}-1\right) \Re f(\omega, t)=0
$$

on $S^{n-1} \times \mathbb{D}_{t}$ only depending on $t$, that is, a one-dimensional equation. It is well known, see [32, Example 3.9.7], that this irregular-singular equation has two pure hyperfunction solutions which we try to recover by an asymptotic series ansatz. We first calculate

$$
\begin{aligned}
t^{2} \frac{\mathrm{d}}{\mathrm{d} t} \delta^{(n)}(t) & =\left[\frac{-1}{2 \pi i} \frac{\tau^{2}(-1)^{n+1}(n+1) !}{\tau^{n+1}}\right] \\
& =(n+1) n\left[\frac{-1}{2 \pi i} \frac{(-1)^{n-1}(n-1) !}{\tau^{n-1}}\right] \\
& = \begin{cases}0 & \text { for } n \leq 1, \\
(n+1) n \cdot \delta^{(n-1)}(t) & \text { otherwise }\end{cases}
\end{aligned}
$$


where we used the usual notation for hyperfunctions in terms of defining functions. With that the equation becomes

$$
\begin{aligned}
\left(t^{2} \frac{\mathrm{d}}{\mathrm{d} t}-1\right) \sum_{n=0}^{\infty} d_{n} \delta^{(n)}(t) & =\sum_{n=1}^{\infty} d_{n}(n+1) n \delta^{(n-1)}(t)-\sum_{n=0}^{\infty} d_{n} \delta^{(n)}(t) \\
& =\sum_{n=1}^{\infty}\left(d_{n}(n+1) n-d_{n-1}\right) \delta^{(n-1)}(t)=0,
\end{aligned}
$$

yielding the recursive prescription $d_{n}=d_{n-1} /(n(n+1))$ for the coefficients. We solve it with initial condition $d_{0}=1$ through setting $d_{n}=((n+1) ! n !)^{-1}$, for all $n=0,1, \ldots$ These coefficients decay fast enough to turn $J=\sum_{n=0}^{\infty} d_{n} D_{t}^{n}$ into a local operator and thus the asymptotic series in this case actually converges to a hyperfunction with support in the single point $\{0\}$. This yields the first hyperfunction solution

$$
\begin{aligned}
f_{1}(\omega, t) & =\sum_{n=0}^{\infty} d_{n} \delta^{(n)}(t) \\
& =\frac{1}{2 \pi i}\left[\sum_{n=0}^{\infty} \frac{(-1)^{n+1}}{(n+1) ! \tau^{n+1}}\right]_{\tau=t}=\frac{1}{2 \pi i}\left[\mathrm{e}^{-1 / \tau}-1\right]_{\tau=t}=\frac{1}{2 \pi i}\left[\mathrm{e}^{-1 / \tau}\right]_{\tau=t} .
\end{aligned}
$$

In this case, the inverse Radon transform exists and is unique, see [60, Theorem 2.4]. It can be explicitly calculated and seen to fulfill the original differential equation. The second hyperfunction solution can be recovered by the following trick: changing the sign of the $G_{-}$-part of the canonical defining function for $-2 \pi i \delta(t)=\left[G_{+}\left(\tau_{+}\right), G_{-}\left(\tau_{-}\right)\right]$, where $G_{+}(\tau)=G_{-}(\tau)=1 / \tau$, essentially gives the Cauchy principal value, that is, the finite part distribution associated with $1 / t$ and likewise for the higher derivatives. We therefore consider the new ansatz

$$
f_{2}(\omega, t)=\sum_{n=0}^{\infty} h_{n} \mathrm{f} \cdot \mathrm{p} \cdot \frac{1}{t^{n}}
$$

(where f.p. denotes Hadamard's finite part, and with the convention f.p. $1=1$ ). Inserting

$$
t^{2} \frac{\mathrm{d}}{\mathrm{d} t} \mathrm{f} \cdot \mathrm{p} \cdot \frac{1}{t^{n+1}}=-(n+1) \mathrm{f} \cdot \mathrm{p} \cdot \frac{1}{t^{n}}
$$

yields

$$
\left(t^{2} \frac{\mathrm{d}}{\mathrm{d} t}-1\right) \sum_{n=0}^{\infty} h_{n} \mathrm{f} \cdot \mathrm{p} \cdot \frac{1}{t^{n+1}}=-h_{0}-\sum_{n=1}^{\infty}\left(h_{n}(n+1)+h_{n-1}\right) \mathrm{f} \cdot \mathrm{p} \cdot \frac{1}{t^{n}}=0 .
$$

With the initial condition $h_{0}=1$ we get $h_{n}=(-1)^{n} /(n+1) !, n=0,1, \ldots$ On subtracting the constant hyperfunction 1 to compensate the term $h_{0}$, we get the second hyperfunction solution by a converging series of defining functions

$$
f_{2}(\omega, t)=1+\sum_{n=1}^{\infty} \frac{(-1)^{n}}{n !} \mathrm{f} \cdot \mathrm{p} \cdot \frac{1}{t^{n}}=\frac{1}{2}\left(\mathrm{e}^{-1 /(t+i 0)}+\mathrm{e}^{-1 /(t-i 0)}\right)
$$


These two hyperfunction solutions span, together with the classical solution $f_{3}(t)=\mathrm{e}^{-1 / t}$, $t>0$, continued by 0 to $(-\infty, 0]$, the solution space of the original equation.

\section{Appendix}

\section{A. Proof of the assertions of Section 2}

A.1. Proof of Proposition 2.4. We refer to [14, 30] for the essentials of locally convex spaces defined by limits of inductive or projective sequences. For the corresponding results in the context of ultradistributions see $[43,44]$. To show that $O_{ \pm \infty}(K)$ is DFS, respectively, that $O_{ \pm \infty}(W)$ is FS for every compactum $K \subset \mathbb{Q}^{\mathbf{n}}$, respectively, for every open set $W \subset \mathbb{Q}^{\mathbf{n}}$, it is enough to show the following lemma.

Lemma A.1. The natural inclusion mapping $\rho: \overline{\mathrm{O}}_{m}^{B}(L) \hookrightarrow \overline{\mathrm{O}}_{n}^{B}(K)$ is a compact mapping whenever $m<n$ and $K \Subset L \subset \mathbb{Q}^{\mathbf{n}}$ are compact.

Proof. Let $\left\{f_{q}\right\} \subset \overline{\mathrm{O}}_{m}^{B}(L)$ be a bounded sequence. Then, we have the estimate

$$
\sup _{z \in U}\left|f_{q}(z)\right| \leq M_{S}{\overline{\left\|f_{q}\right\|_{n, K}}} \leq N_{S}{\overline{\left\|f_{q}\right\|_{m, L}}}
$$

which holds, with constants $M_{S}, N_{S}>0$, for every compact set $S \subset K_{\mathbb{C}|\mathbf{n}|}^{\circ}$, every open neighborhood $U$ of $S$ in $K_{\mathbb{C}^{|n|}}$, and uniformly in $q$. Under these conditions, [20, Corollary 2.2.5] implies that there is a subsequence $f_{q_{k}}$ converging to a limit $f \in \mathcal{O}\left(K_{\mathbb{C}^{|\mathbf{n}|}}^{\circ}\right)$ uniformly on compact subsets of $K_{\mathbb{C}|\mathbf{n}|}^{\circ}$. The second inequality above shows that $f_{q_{k}}$ converges to $f$ in $\overline{\mathrm{O}}_{n}^{B}(K)$. This shows the assertion.

To show nuclearity of the four types of spaces in question, we proceed as in the proof of [34, Proposition 2.12] or [22, Proposition 2.1.3], by first showing nuclearity of yet another space. Let $U \subset \mathbb{Q}^{\mathbf{n}}$ be open and define for every integer $s$ the Fréchet space

$$
\widehat{O}_{\underline{s}}(U) \stackrel{\text { def }}{=}\left\{f \in O_{(}\left(U_{\mathbb{C}|\mathbf{n}|}\right) \mid\|f\|_{s, p, K}<\infty, K \subset U \text { compact, } p=2,3, \ldots\right\},
$$

with the following system of seminorms for $p \geq 2$ :

$$
\|f\|_{s, p, K} \stackrel{\text { def }}{=} \int_{K_{\mathbb{C}|\mathbf{n}|}}|f(z)| M_{p}(z) \mathrm{d} \lambda(z) \quad \text { where } M_{p}(z)=(1+|\operatorname{Re} z|)^{-s-|s| / p} .
$$

Here, $\lambda$ denotes the Lebesgue measure on $\mathbb{C}^{|\mathbf{n}|}$.

Lemma A.2. The space $\mathrm{O}_{\underline{\underline{s}}}(U)$ is nuclear.

Proof. Repeat the proof of [34, Proposition 2.11], or [22, Lemma 2.1.4] with minor modifications.

Now, choose a fundamental system $\left\{U_{m}\right\}_{m \in \mathbb{N}}$ of open neighborhoods of $K$ such that $U_{m+1} \Subset U_{m}$ and further an exhausting sequence $\left\{\overline{\overline{V_{m}}}\right\}_{m \in \mathbb{N}}$ for $W$ such that $V_{m} \Subset V_{m+1}$ are open sets in $W$. 
Lemma A.3. There are linear, topological isomorphisms

$$
\mathrm{O}_{\infty}(K) \cong \underline{\lim } \hat{O}_{\underline{m}}\left(U_{m}\right), \quad \mathrm{O}_{-\infty}(W) \cong \lim _{\underline{-m}}\left(V_{m}\right) .
$$

Proof. We have a continuous inclusion ${\overline{O^{\prime}}}_{m}^{B}\left(\overline{\overline{U_{m}}}\right) \hookrightarrow O_{m+|\mathbf{n}|}\left(U_{m+|\mathbf{n}|}\right)$. On the other hand, an application of Cauchy's integral formula as in the proof of [22, Lemma 2.1.4] yields an embedding $\widehat{O}_{\underline{m}}\left(U_{m}\right) \hookrightarrow \overline{\mathrm{O}}_{m+1}^{B}\left(\overline{\overline{U_{m+1}}}\right)$. This suffices to show equivalence of the inductive limits in the tempered case. The asymptotic case follows by similar considerations.

The permanence properties of nuclearity, see [61, Proposition 50.1], then imply all assertions of Proposition 2.4.

A.2. Proof of Proposition 2.5. Since the proof is mainly an application of general facts about locally convex spaces and their tensor products, we refer the reader to the proofs of [22, Proposition 2.1.7-2.1.10], which in turn follows [34, Proposition 3.6], and merely note the only modification that has to be inserted: there is an auxiliary space $\mathscr{E}_{*}$ of $C^{\infty}$ functions which has to be replaced by

$$
\begin{aligned}
& \mathscr{\mathscr { E }}_{-\infty}(U) \stackrel{\text { def }}{=}\left\{f \in C^{\infty}\left(U_{\mathbb{C}^{|\mathbf{n}|}}\right)\left|\forall \gamma>0: \sup _{z \in K_{\mathbb{C}}|\mathbf{n}|}\right| f^{(\alpha)}(z)(1+|z|)^{\gamma} \mid<\infty\right\}, \\
& \mathscr{E}_{\infty}(U) \stackrel{\text { def }}{=}\left\{f \in C^{\infty}\left(U_{\mathbb{C}^{|\mathbf{n}|}}\right)\left|\exists \gamma \in \mathbb{R}: \sup _{z \in K_{\mathbb{C}|\mathbf{n}|}}\right| f^{(\alpha)}(z)(1+|z|)^{\gamma} \mid<\infty\right\}
\end{aligned}
$$

for the asymptotic and tempered case of the assertions, respectively, and any open subset $U$ of $\mathbb{Q}^{\mathbf{n}}$. The conditions in the definitions above are meant to hold for every compact set $K \subset U$ and every real partial derivative $f^{(\alpha)}, \alpha \in \mathbb{N}_{0}^{2|\mathbf{n}|}$. The proof can then be carried out as in the reference indicated above, using further the results [61, Theorem 39.2 and Proposition 36.1], [21, Corollary 1 of Lemma A], and [1, Theorem 5 of Section 4].

A.3. Proof of Theorem 2.6. This proof follows [22, Theorem 2.1.14]. It heavily depends on a notion originally designed for slowly increasing, that is, infraexponential functions in the context of Fourier hyperfunctions, see [29], but which is also applicable in our case due to its mainly geometric nature.

Definition A.4 [34, Definition 5.1]. An open subset $V$ of $\mathbb{Q}^{\mathbf{n}}$ is called an $\mathbf{O}^{*}$-pseudoconvex set if

(i) $\sup \left\{\left|\operatorname{Im} z^{\prime \prime}\right|,\left|\operatorname{Im} z^{\prime \prime \prime}\right|-\left|\operatorname{Re} z^{\prime \prime \prime}\right| \mid z \in V_{\mathbb{C}|\mathbf{n}|}\right\}<\infty$,

(ii) there exists a smooth, plurisubharmonic function $\varphi(z)$ on $V_{\mathbb{C}|\mathbf{n}|}$ which is bounded on $L_{\mathbb{C}|\mathbf{n}|}$ for every compactum $L \subset V$, and such that $\overline{V_{c}}$ is compact in $V$, where $V_{c} \stackrel{\text { def }}{=}\left\{z \in v_{\mathbb{C}|\mathbf{n}|} \mid \varphi(z)<c\right\}$.

Condition (i) only states that a pseudoconvex domain is of finite width around $\mathbb{D}^{\mathbf{n}}$ in the second part of the coordinates $\mathbf{n}=\left(n_{1}, n_{2}, n_{3}\right)$, and of finite inclination above $\mathbb{D}^{\mathbf{n}}$ in 
the third part. The second condition is a direct generalization of the notion of pseudoconvexity in the complex domain, see [20, Theorem 2.6.7]. The following is an analogue of Grauert's theorem.

Theorem A.5 (see [34, Theorem 5.3], [22, Theorem 2.1.13]). For every open $S \subset \mathbb{D}^{\mathbf{n}}$ and an open neighborhood $U \subset \mathbb{Q}^{\mathbf{n}}$ of $S$, there exists an $0^{*}$-pseudoconvex set $V \subset U$ such that $S=V \cap \mathbb{D}^{\mathbf{n}}$.

Thus the compact set $K$ in the statement of Theorem 2.6 has a fundamental system of neighborhoods in $\mathbb{Q}^{\mathbf{n}}$ consisting of $\mathrm{O}^{*}$-pseudoconvex sets. Therefore, we have only to prove that $\mathrm{H}_{U}^{1}\left(\mathbb{Q}^{\mathbf{n}} ; \mathbb{O}_{ \pm \infty}\right)=0$ for every $\mathbb{O}^{*}$-pseudoconvex set $U \subset \mathbb{Q}^{\mathbf{n}}$, such that $\left|\operatorname{Im} z^{\prime}\right|<$ $\varepsilon,\left|\operatorname{Im} z^{\prime \prime}\right|<\varepsilon$, and $\left|\operatorname{Im} z^{\prime \prime \prime}\right|<\varepsilon\left(1+\left|\operatorname{Re} z^{\prime \prime \prime}\right|\right)$ on $U_{\mathbb{C}|\mathbf{n}|}$ for some $\varepsilon$ sufficiently small for our later purposes. Since $U$ is paracompact, the relative cohomology groups coincide with the Cech cohomology groups and it remains only to show $\mathrm{H}^{1}\left(\left\{U_{j}\right\}_{j \in \mathbb{N}} ; \bigcirc_{ \pm \infty}\right)=0$ for any locally finite covering $\left\{U_{j}\right\}_{j \in \mathbb{N}}$ of $U$ such that $V_{j}=U_{j \mathbb{C}|\mathbf{n}|}$ is $O^{*}$-pseudoconvex.

We prove the assertion for $\mathrm{O}_{\infty}$ only, since a very similar argument applies in the case of asymptotic functions. Let $C^{s}\left(Z^{\operatorname{loc}}\left(\left\{V_{j}\right\}\right)\right)$ be the space of cochains $c=\left\{c_{J} \mid J=\left(j_{0}, \ldots, j_{s}\right) \in\right.$ $\mathbb{N}^{s+1}$ s such that

(i) $\bar{\partial} c_{J}=0$ on $V_{J}=V_{j_{0}} \cap \cdots \cap V_{j_{s}}$,

(ii) $\sum_{J \in M} \int_{V_{J}}\left|c_{J}\right|^{2} \mathrm{~d} \lambda<\infty$, for any finite subset $M$ of $\mathbb{N}^{s+1}$.

If $d=\left\{d_{i j}\right\}$ represents a cocycle in $\mathrm{H}^{1}\left(\left\{U_{j}\right\}_{j \in \mathbb{N}} ; O_{\infty}\right)$, then there is a $\gamma>0$ such that $\left.d_{i j} \cdot j_{-\gamma}\right|_{V_{i j}}$ is in $C^{1}\left(Z^{\text {loc }}\left(\left\{V_{j}\right\}\right)\right)$, where the multiplier $j_{\gamma}$ is defined for $\gamma \in \mathbb{R}$ by $j_{\gamma} \stackrel{\text { def }}{=}(1+$ $\left.z^{\prime \prime 2}+z^{\prime \prime \prime 2}\right)^{\gamma / 2}$. Here, we assume that the $\varepsilon$ above is small enough such that $j_{-\gamma}$ and $j_{-\gamma-2}$ (to be used below) are holomorphic on $U_{\mathbb{C}^{\mid \mathbf{n}}}$, that is, we implicitly consider the simultaneous inductive limit over neighborhoods $U$ of $K$ and increasing growth order of forms in $\mathrm{H}^{1}\left(U ; \mathrm{O}_{\infty}\right)$. Define $c=\left\{c_{i j}\right\} \in C^{1}\left(Z^{\operatorname{loc}}\left(\left\{V_{j}\right\}\right)\right)$ such that $\delta c=0$, by $\left.c_{i j} \stackrel{\text { def }}{=} d_{i j} \cdot j_{-\gamma-2}\right|_{V_{i j}}$, where $\delta$ is the coboundary operator. Let $\left\{\chi_{j}\right\}$ be a smooth partition of unity subordinate to $\left\{V_{j}\right\}$, and set $b_{j} \stackrel{\text { def }}{=} \sum_{i} \chi_{i} c_{i j}$. Then $\delta c=0 \Rightarrow \delta b=c$ and consequently $\delta \bar{\partial} b=\bar{\partial} c=0$ by condition (i). Since $\sum \chi_{i}=1$ and $\chi_{i} \geq 0$, we have

$$
\int_{V_{j}}\left|b_{j}\right|^{2} \mathrm{~d} \lambda \leq \sum_{i} \int_{V_{i}} \chi_{i}\left|c_{i j}\right|^{2} \mathrm{~d} \lambda<\infty
$$

by the triangle inequality and condition (ii). Since $U$ is $0^{*}$-pseudoconvex, there exists a smooth plurisubharmonic function $\psi$ on $V \stackrel{\text { def }}{=} U_{\mathbb{C}|\mathbf{n}|}$ that satisfies the two conditions (1) $\sum_{j}\left|\bar{\partial} \chi_{j}(z)\right| \leq \exp (\psi(z))$ and (2) $\sup _{K_{\mathbb{C}|\mathbf{n}|}} \psi \leq C_{K}$ for every relatively compact subset $K$ of $U$. Thus, (1) and condition (ii) above, together with the definition of $c$ and $b$ imply

$$
\sum_{j \in M} \int_{V_{j}}\left|\bar{\partial} b_{j}\right|^{2}\left(1+|z|^{2}\right)^{2} \exp (-\psi(z)) \mathrm{d} \lambda<\infty
$$

for all finite sets $M \subset \mathbb{N}$. Since $\bar{\partial} b$ is closed, it defines a global section $f$ on $U_{\mathbb{C}^{|\mathbf{n}|}}$. This and the last estimate allow us to make use of [20, Theorem 4.4.2], and conclude that there 
exists a smooth section $u$ on $U_{\mathbb{C}^{|\mathbf{n}|}}$ such that $\bar{\partial} u=f$, and with $\int_{K_{\mathbb{C}}|\mathbf{n}|}|u|^{2} \mathrm{~d} \lambda<\infty$ for all relatively compact subsets $K$ of $U$ (this theorem increases the growth order by two, but remember the estimate on the integral over $\left|\bar{\partial} b_{j}\right|$ above). Set $c_{j}^{\prime} \stackrel{\text { def }}{=} b_{j}-\left.u\right|_{V_{j}}$. Then $\bar{\partial} c_{j}^{\prime}=0$, $\delta c^{\prime}=\delta b=c$, and $c^{\prime}$ is an element of $C^{1}\left(Z^{\operatorname{loc}}\left(\left\{V_{j}\right\}\right)\right)$. Finally, with $\left.d_{j}^{\prime} \stackrel{\text { def }}{=} c_{j}^{\prime} \cdot j_{\gamma+2}\right|_{V_{j}}$ we find that the collection $d^{\prime} \stackrel{\text { def }}{=}\left\{d_{j}^{\prime}\right\}$ is a subset of $O_{\infty}$ such that $\delta d^{\prime}=d$, which means that $d=0$ in $\mathrm{H}^{1}\left(\left\{U_{j}\right\}_{j \in \mathbb{N}} ; \mathrm{O}_{\infty}\right)$. Since $d$ was a generic element, we find $\mathrm{H}^{1}\left(\left\{U_{j}\right\}_{j \in \mathbb{N}} ; \mathrm{O}_{\infty}\right)=0$.

A.4. Proof of Theorem 2.8. The proof follows the original one in the case of Fourier hyperfunctions [29, Theorem 2.2.1], see also [34, proof of Theorem 3.1] and [22, Section 2.2].

Again, we consider only the case $\mathcal{P}_{\infty}$. Since $\mathbb{D}^{\mathbf{n}}$ is $\sigma$-compact, the assertion of the theorem factorizes in the first and the last two variables and it is sufficient to prove.

Theorem A.6. If $L=\left\{x^{\prime} \in \mathbb{R}^{n_{1}}|| x^{\prime} \mid \leq a\right\} \times \mathbb{D}^{n_{2}} \times \mathbb{D}^{n_{3}}$ contains the compact set $K \subset \mathbb{D}^{\mathbf{n}}$ for some $a>0$, then $\mathcal{P}_{\infty}(L)$ is dense in $\mathcal{P}_{\infty}(K)$.

For $W \subset \mathbb{Q}^{\mathbf{n}}$ open and $\eta>0$, we define $\mathscr{O}_{\eta}^{2, \text { loc }}(W)$ to be the space of all holomorphic functions on $W_{\mathbb{C}|\mathbf{n}|}$ such that for all compacta $K \subset W$ holds $\int_{K_{\mathbb{C}|\mathbf{n}|}}|f|^{2}\left(1+\left|z^{\prime \prime}\right|+\right.$ $\left.\left|z^{\prime \prime \prime}\right|\right)^{-\eta} \mathrm{d} \lambda<\infty$. This space is an FS-space (consider any exhaustive sequence of compacta for $W$ ), and we have the following.

LEMmA A.7. If $\left\{W_{j}\right\}$ is a fundamental system of neighborhoods for a compact subset $K$ in $\mathbb{Q}^{\mathbf{n}}$, then there is a linear topological isomorphism

$$
\mathrm{O}_{\infty}(K) \cong \lim _{j}^{2, \text { loc }}\left(W_{j}\right)
$$

To see this, note that for sufficiently large $j$, there are numbers $k, l$ such that there exist continuous inclusions $\overline{\mathrm{O}}_{j}^{B}\left(W_{j}\right) \hookrightarrow \mathcal{O}_{j+k}^{2 \text { loc }}\left(W_{j+k}\right)$ and $\widehat{O}_{j+k}^{2, \text { loc }}\left(W_{j+k}\right) \hookrightarrow \overline{\mathrm{O}}_{j+k+l}^{B}\left(W_{j+k+l}\right)$.

Now, let $\left\{W_{j}\right\}$ and $\left\{V_{j}\right\}$ be fundamental systems of neighborhoods for the compact sets $L \subset K \subset \mathbb{D}^{\mathbf{n}}$ of Theorem A.6, respectively, such that $V_{j} \subset W_{j}$ for all $j$. Then $\mathrm{O}_{\infty}(L) \cong \underline{\lim }^{2} \hat{O}_{j}^{2, \text { loc }}\left(W_{j}\right)$ and $\mathrm{O}_{\infty}(K) \cong \underline{\lim }^{2} \hat{O}_{j}^{2, \text { loc }}\left(V_{j}\right)$. We are done if we are able to show that $O_{j}^{2 \text {,loc }}\left(W_{j}\right)$ is dense in $O_{j}^{2, \text { loc }}\left(V_{j}\right)$ for sufficiently large $j$ (cf. [22, Lemma 2.2.7]). For this, it trivially suffices to show that $O_{l}^{2, \text { loc }}\left(W_{j}\right)$ is dense in $O_{j}^{2, \text { loc }}\left(V_{j}\right)$ for $l \leq j$ sufficiently large. Setting for brevity $W=W_{j}, V=V_{j}$, the Hahn-Banach theorem tells us that we only need to prove the following: if $\mu \in \mathcal{O}_{j}^{2, \text { loc }}(V)^{\prime}$ and $\langle\mu, v\rangle=0$ for all $v \in \mathcal{O}_{l}^{2, \text { loc }}(W)$, then $\mu=0$.

The solution of our problem requires finding solutions of the dual Cauchy-Riemann differential equation with growth conditions. These solutions traditionally live in $L^{2}$ spaces, and so we have to make another definition: $L_{\eta}^{2, \text { loc }}(W)$ is the space of locally square integrable functions on $W_{\mathbb{C}|\mathbf{n}|}$ that satisfy the same integrability condition as the functions in $O_{\eta}^{2, \text { loc }}(W) \cdot L_{\eta}^{2, \text { loc }}(W)$ is a $F S^{*}$-space and $O_{\eta}^{2, \text { loc }}(W)$ is a closed subspace of it. The dual space of $L_{\eta}^{2, \text { loc }}(W)$ is the space $L_{-\eta}^{2, \mathrm{c}}(W)$ of functions $f \in L^{2, \text { loc }}\left(W_{\mathbb{C}|\mathbf{n}|}\right)$ with compact support in $W$, and such that $\int_{W_{\mathbb{C}|\mathbf{n}|}}|f|^{2}\left(1+\left|z^{\prime \prime}\right|+\left|z^{\prime \prime \prime}\right|\right)^{\eta} \mathrm{d} \lambda<\infty$. 
Now, since $O_{j}^{2, \text { loc }}(V)$ is a closed subspace of $L_{j}^{2, \text { loc }}(V)$, there exists a representative $u \in$ $L_{-j}^{2, \mathrm{c}}(V)$ of $\mu \in \mathbb{O}_{j}^{2, \text { loc }}(V)^{\prime}$, that is,

$$
\langle\mu, v\rangle=\int_{V_{\mathbb{C}|\mathbf{n}|}} v \bar{u} \mathrm{~d} \lambda \quad \text { for } v \in \mathbb{O}_{j}^{2, \text { loc }}(V)
$$

Thus we only have to prove that $u$ is orthogonal to $O_{j}^{2, \text { loc }}(V)$.

Set $T=\overline{\overline{\operatorname{supp}(u)}}$. By [22, Lemma 2.2.6], there exists a neighborhood $U$ of $T$ which is relatively compact in $V$, and a strictly plurisubharmonic smooth function $\theta$ on $W_{\mathbb{C}^{|\mathbf{n}|}}$ such that (i) $\theta(z)<0$ on $T_{\mathbb{C}^{\mid \mathbf{n}} \mid}$, (ii) $\theta(z)>0$ on a neighborhood $N$ of $(\partial U)_{\mathbb{C}^{\mid \mathbf{n}} \mid}$, and (iii) $\sup _{L_{\mathbb{C}|\mathbf{n}|}} \theta(z)<\infty$ for any relatively compact set $L \subset W$. Then, [19, Theorem 2.3.2] ensures that there exists a form $f \in L_{2}^{(0,1)}\left(W_{\mathbb{C}|\mathbf{n}|} ;(2-j) \log (1+|z|)\right)$ such that $u=\vartheta f$, where $\vartheta$ is the adjoint of the $\partial$-operator. Furthermore, $\operatorname{supp}(f) \subset\left\{z \in W_{\mathbb{C}|\mathbf{n}|} \mid \theta(z) \leq 0\right\}$. Choose a smooth function $\chi$ on $W_{\mathbb{C}^{|\mathbf{n}|}}$ such that $0 \leq \chi(z) \leq 1, \chi(z)=1$ on $T_{\mathbb{C}^{|\mathbf{n}|}}, \chi(z)=0$ on $\overline{(U \cup N)_{\mathbb{C}^{|\mathbf{n}|}}}, \operatorname{supp}(\bar{\partial} \chi) \subset N$, and $\sup |\bar{\partial} \chi|<\infty$. Then, for every $w \in \mathbb{O}_{j-2}^{2, \text { loc }}(V)$ holds

$$
\langle\mu, w\rangle=\int_{V_{\mathbb{C}|\mathbf{n}|}} w \bar{u} \mathrm{~d} \lambda=\int_{W_{\mathbb{C}|\mathbf{n}|}}(\chi w) \bar{u} \mathrm{~d} \lambda=\int_{W_{\mathbb{C}|\mathbf{n}|}}(\chi w) \bar{\vartheta} \bar{f} \mathrm{~d} \lambda=\int_{W_{\mathbb{C}}|\mathbf{n}|} \bar{\partial}(\chi w) \bar{f} \mathrm{~d} \lambda=0
$$

by the constraints on $f$ and $\chi$. A trivial analogue of [22, Lemma 2.2.5] implies that $\mathrm{O}_{j-2}^{2, \text { loc }}(V)$ is dense in $\mathrm{O}_{j}^{2, \text { loc }}(V)$ if we choose the shape of $V$ as in [22, page 234]. This completes the proof of Theorem A.6.

\section{Acknowledgments}

This research was supported by the Deutsche Forschungsgemeinschaft DFG (http://www. dfg.de). The author is very grateful for the help of and the discussions with E. Brüning, F. Constantinescu, R. Estrada, and S. Nagamachi. The hospitality of the Department of Mathematics and Applied Mathematics, University of Durban-Westville, South Africa, and the Dipartimento di Fisica E. Fermi (http://www.df.unipi.it), Università di Pisa, and the INFN (http://www.infn.it), sezione Pisa, Italy, is gratefully acknowledged

\section{References}

[1] K.-D. Bierstedt and R. Meise, Lokalkonvexe Unterräume in topologischen Vektorräumen und das $\epsilon$-Produkt, Manuscripta Math. 8 (1973), 143-172.

[2] E. Brüning and S. Nagamachi, Hyperfunction quantum field theory: basic structural results, J. Math. Phys. 30 (1989), no. 10, 2340-2359.

[3] Closure of field operators, asymptotic abelianness, and vacuum structure in hyperfunction quantum field theory, J. Math. Phys. 39 (1998), no. 10, 5098-5111.

[4] E. Brüning and P. Stichel, On the equivalence of scaling, light-cone singularities and asymptotic behaviour of the Jost-Lehmann spectral function, Comm. Math. Phys. 36 (1974), 137-156.

[5] _ Asymptotics and light-cone singularities in quantum field theory, International Symposium on Mathematical Problems in Theoretical Physics (Kyoto Univ., Kyoto, 1975) (H. Araki, ed.), Lecture Notes in Phys., vol. 39, Springer, Berlin, 1975, pp. 72-84. 
[6] S.-Y. Chung, D. Kim, and E. G. Lee, Schwartz kernel theorem for the Fourier hyperfunctions, Tsukuba J. Math. 19 (1995), no. 2, 377-385.

[7] I. Cioranescu, Moment sequences for ultradistributions, Math. Z. 204 (1990), no. 3, 391-400.

[8] F. Constantinescu and W. Thaleimer, Euclidean Green's functions for Jaffe fields, Comm. Math. Phys. 38 (1974), no. 2, 299-316.

[9]__L Ultradistributions and quantum fields: Fourier-Laplace transforms and boundary values of analytic functions, Rep. Math. Phys. 16 (1979), no. 2, 167-180.

[10] A. L. Durán and R. Estrada, Strong moment problems for rapidly decreasing smooth functions, Proc. Amer. Math. Soc. 120 (1994), no. 2, 529-534.

[11] R. Estrada, J. M. Gracia-Bondía, and J. C. Várilly, On summability of distributions and spectral geometry, Comm. Math. Phys. 191 (1998), no. 1, 219-248.

[12] R. Estrada and R. P. Kanwal, A distributional theory for asymptotic expansions, Proc. Roy. Soc. London Ser. A 428 (1990), no. 1875, 399-430.

[13] - Asymptotic Analysis. A Distributional Approach, Birkhäuser, Massachusetts, 1994.

[14] K. Floret and J. Wloka, Einführung in die Theorie der Lokalkonvexen Räume, Lecture Notes in Mathematics, no. 56, Springer, Berlin, 1968.

[15] I. M. Gel'fand and G. E. Shilov, Generalized functions. Vol. 2. Spaces of fundamental and generalized functions, Academic Press, New York, 1968, Translated from the Russian by Morris D. Friedman, Amiel Feinstein and Christian P. Peltzer.

[16] A. Grossmann, G. Loupias, and E. M. Stein, An algebra of pseudodifferential operators and quantum mechanics in phase space, Ann. Inst. Fourier (Grenoble) 18 (1968), no. fasc. 2, 343-368.

[17] M. Hasumi, Note on the n-dimensional tempered ultra-distributions, Tôhoku Math. J. (2) 13 (1961), 94-104.

[18] S. Helgason, Groups and Geometric Analysis Integral Geometry, Invariant Differential Operators, and Spherical Functions, Pure and Applied Mathematics, vol. 113, Academic Press, Florida, 1984.

[19] L. Hörmander, $L^{2}$ estimates and existence theorems for the $\bar{\partial}$ operator, Acta Math. 113 (1965), 89-152.

[20] - An Introduction to Complex Analysis in Several Variables, D. Van Nostrand, London, 1966.

[21] Y. Ito, On the theory of vector-valued hyperfunctions, J. Math. Tokushima Univ. 13 (1979), 2951.

[22] - Fourier hyperfunctions of general type, J. Math. Kyoto Univ. 28 (1988), no. 2, 213-265.

[23] - Vector valued Fourier hyperfunctions, J. Math. Kyoto Univ. 32 (1992), no. 2, 259-285.

[24] L. Jakóbczyk and F. Strocchi, Euclidean formulation of quantum field theory without positivity, Comm. Math. Phys. 119 (1988), no. 4, 529-541.

[25] K. Junker, Vektorwertige Fourierhyperfunktionen, Diplomarbeit, Universität Düsseldorf, 1978.

[26] A. Kaneko, Introduction to Hyperfunctions, Mathematics and its Applications (Japanese Series), vol. 3, Kluwer Academic Publishers Group, Dordrecht, 1988.

[27] M. Kashiwara and T. Kawai, Second-microlocalization and asymptotic expansions, Complex Analysis, Microlocal Calculus and Relativistic Quantum Theory (Proc. Internat. Colloq., Centre Phys., Les Houches, 1979) (D. Iagolnitzer, ed.), Lecture Notes in Phys., vol. 126, Springer, Berlin, September 1980, pp. 21-76.

[28] M. Kashiwara, T. Kawai, and T. Kimura, Foundations of Algebraic Analysis, Princeton Mathematical Series, vol. 37, Princeton University Press, New Jersey, 1986.

[29] T. Kawai, On the theory of Fourier hyperfunctions and its applications to partial differential equations with constant coefficients, J. Fac. Sci. Univ. Tokyo Sect. IA Math. 17 (1970), 467-517.

[30] H. Komatsu, Ultradistributions. I. Structure theorems and a characterization, J. Fac. Sci. Univ. Tokyo Sect. IA Math. 20 (1973), 25-105. 

, Microlocal analysis in Gevrey classes and in complex domains, Microlocal Analysis and Applications (Montecatini Terme, 1989), Lecture Notes in Math., vol. 1495, Springer, Berlin, 1991, pp. 161-236, 2nd session of the Centro Internazionale Matematico Estivo (C.I.M.E.), Montecattini Terme, Italy.

[32] M. Morimoto, An Introduction to Sato'S Hyperfunctions, Translations of Mathematical Monographs, vol. 129, American Mathematical Society, Rhode Island, 1993.

[33] U. Moschella and F. Strocchi, The choice of test functions in gauge quantum field theories, Lett. Math. Phys. 24 (1992), no. 2, 103-113.

[34] S. Nagamachi, The theory of vector valued Fourier hyperfunctions of mixed type. I, Publ. Res. Inst. Math. Sci. 17 (1981), no. 1, 25-63.

[35] - The theory of vector valued Fourier hyperfunctions of mixed type. II, Publ. Res. Inst. Math. Sci. 17 (1981), no. 1, 65-93.

[36] S. Nagamachi and N. Mugibayashi, Hyperfunction quantum field theory, Comm. Math. Phys. 46 (1976), no. 2, 119-134.

[37] Hyperfunction quantum field theory. II. Euclidean Green's functions, Comm. Math. Phys. 49 (1976), no. 3, 257-275.

[38] , Quantum field theory in terms of Fourier hyperfunctions, Publ. Res. Inst. Math. Sci. 12 (1976/1977), suppl 309-341, Proceedings of the Oji Seminar on Algebraic Analysis and the RIMS Symposium on Algebraic Analysis (Kyoto Univ., Kyoto, 1976).

[39] - The Haag-Ruelle formulation of scattering in hyperfunction quantum field theory, Rep. Math. Phys. 16 (1979), no. 2, 181-201.

[40] Hyperfunctions and renormalization, J. Math. Phys. 27 (1986), no. 3, 832-839.

[41] F. W. J. Olver, Asymptotics and Special Functions, AKP Classics, A K Peters, Massachusetts, 1997, Reprint of the 1974 original [Academic Press, New York].

[42] Y. S. Park and M. Morimoto, Fourier ultra-hyperfunctions in the Euclidean n-space, J. Fac. Sci. Univ. Tokyo Sect. IA Math. 20 (1973), 121-127.

[43] H.-J. Petzsche, Die Nuklearität der Ultradistributionsräume und der Satz vom Kern. I, Manuscripta Math. 24 (1978), no. 2, 133-171 (German).

[44] Die Nuklearität der Ultradistributionsräume und der Satz vom Kern. II, Manuscripta Math. 27 (1979), no. 3, 221-251 (German).

[45] - Generalized functions and the boundary values of holomorphic functions, J. Fac. Sci. Univ. Tokyo Sect. IA Math. 31 (1984), no. 2, 391-431.

[46] D. Pierotti, The exponential of the two-dimensional massless scalar field as an infrared Jaffe field, Lett. Math. Phys. 15 (1988), no. 3, 219-230.

[47] Y. Saburi, Fundamental properties of modified Fourier hyperfunctions, Tokyo J. Math. 8 (1985), no. 1, 231-273.

[48] M. Sato, Theory of hyperfunctions. I, J. Fac. Sci. Univ. Tokyo Sect. IA Math. 8 (1959), no. 1, 139-193.

[49] - Theory of hyperfunctions. II, J. Fac. Sci. Univ. Tokyo Sect. IA Math. 8 (1960), no. 2, $387-437$.

[50] A. U. Schmidt, Euclidean reconstruction in quantum field theory: between tempered distributions and Fourier hyperfunctions, Preprint, Johann Wolfgang Goethe-Universit"at, Frankfurt am Main, Germany, http://arXiv.org/abs/math-ph/9811002, April 1997.

[51] Mathematical problems of gauge quantum field theory: a survey of the Schwinger model, Univ. Iagel. Acta Math. (1997), no. 34, 113-134.

[52] _ Asymptotische Hyperfunktionen, temperierte Hyperfunktionen und asymptotische Entwicklungen, Phd dissertation, Johann Wolfgang Goethe-Universität, Berlin, 1999, Frankfurt am Main. , Infinite infrared regularization and a state space for the Heisenberg algebra, J. Math. Phys. 43 (2002), no. 1, 243-259. 
[54] - On Phragmén-Lindelöf principles of type L $L^{p}$, Appl. Math. Notes 3 (2003), 178-182.

[55] J. Sebastião e Silva, Les fonctions analytiques comme ultra-distributions dans le calcul opérationnel, Math. Ann. 136 (1958), 58-96.

[56] M. A. Soloviev, An extension of distribution theory and of the Paley-Wiener-Schwartz theorem related to quantum gauge theory, Comm. Math. Phys. 184 (1997), no. 3, 579-596.

[57] Wick-ordered entire functions of the indefinite metric free field, Lett. Math. Phys. 41 (1997), no. 3, 265-277.

[58] F. Strocchi, Selected Topics on the General Properties of Quantum Field Theory, World Scientific Lecture Notes in Physics, vol. 51, World Scientific Publishing, New Jersey, 1993.

[59] G. 't Hooft, Under the Spell of the Gauge Principle, Advanced Series in Mathematical Physics, vol. 19, World Scientific Publishing, New Jersey, 1994.

[60] T. Takiguchi and A. Kaneko, Radon transform of hyperfunctions and support theorem, Hokkaido Math. J. 24 (1995), no. 1, 63-103.

[61] F. Trèves, Topological Vector Spaces, Distributions and Kernels, Academic Press, New York, 1967.

[62] V. S. Vladimirov, Yu. N. Drozzinov, and B. I. Zavialov, Tauberian Theorems for Generalized Functions, Mathematics and its Applications (Soviet Series), vol. 10, Kluwer Academic Publishers Group, Dordrecht, 1988.

[63] W. Wasow, Asymptotic Expansions for Ordinary Differential Equations, Pure and Applied Mathematics, vol. 14, Interscience Publishers John Wiley \& Sons, London, 1965.

[64] E. T. Whittaker and G. N. Watson, A Course of Modern Analysis, Cambridge University Press, London, 1952.

[65] A. S. Wightman, The choice of test functions in quantum field theory, Mathematical analysis and applications, Part B, Adv. in Math. Suppl. Stud., vol. 7, Academic Press, New York, 1981, pp. 769-791.

[66] Y. Yeom, S.-Y. Chung, and D. Kim, Moment problem for hyperfunctions and heat kernel method, Preprint RIM-GARC 98-8, Seoul National University, Korea, April 1998.

Andreas U. Schmidt: Fraunhofer-Institute for Secure Information Technology, Dolivostrasse 15, 64293 Darmstadt, Germany

E-mail address: aschmidt@math.uni-frankfurt.de 


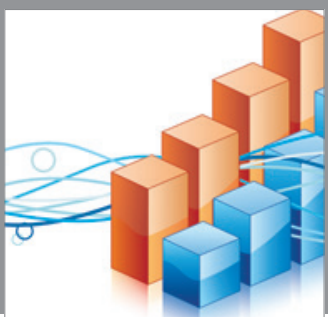

Advances in

Operations Research

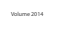

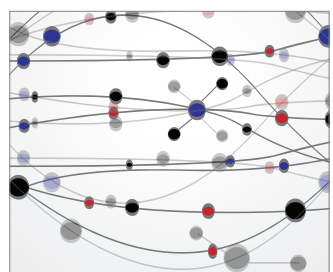

\section{The Scientific} World Journal
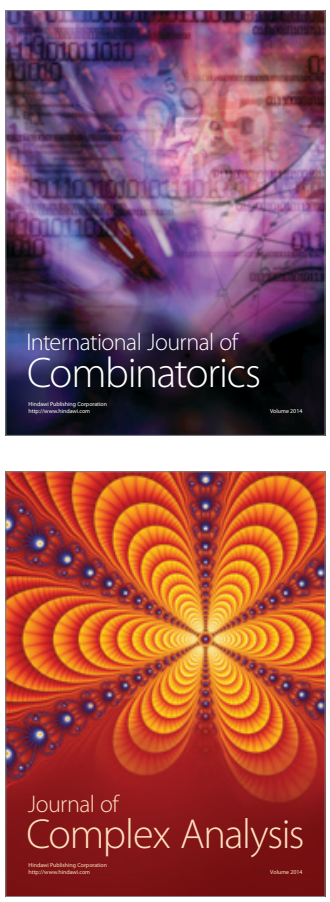

International Journal of

Mathematics and

Mathematical

Sciences
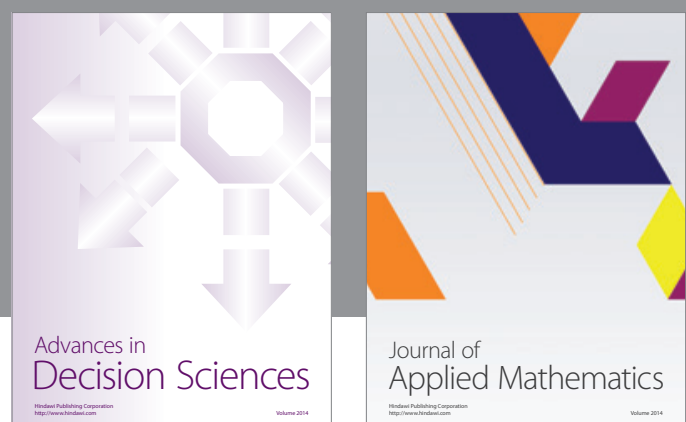

Journal of

Applied Mathematics
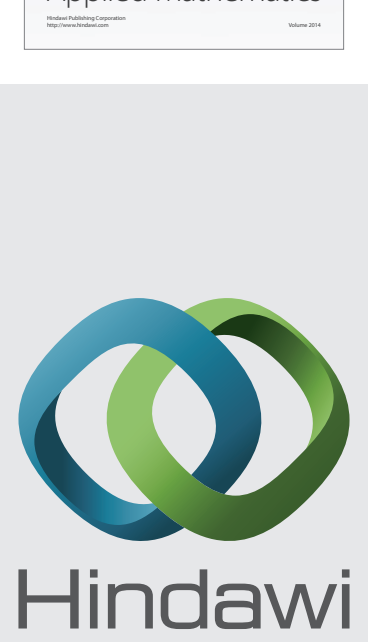

Submit your manuscripts at http://www.hindawi.com
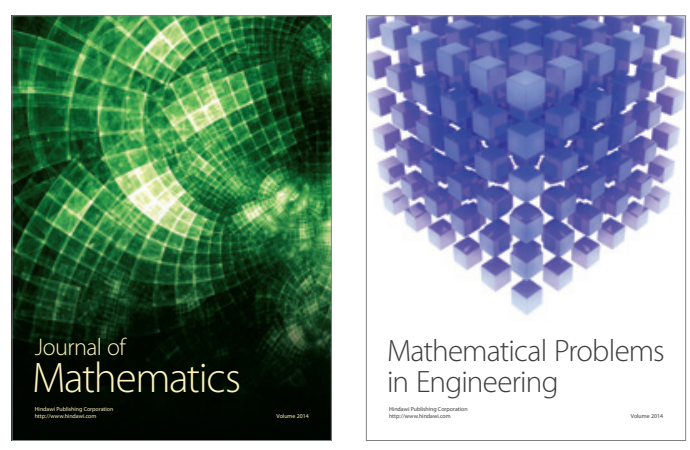

Mathematical Problems in Engineering
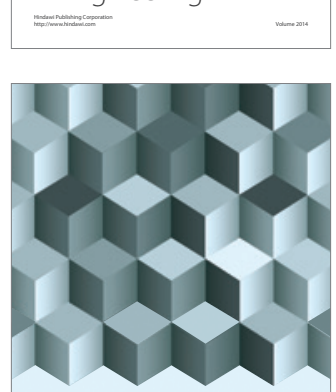

Journal of

Function Spaces
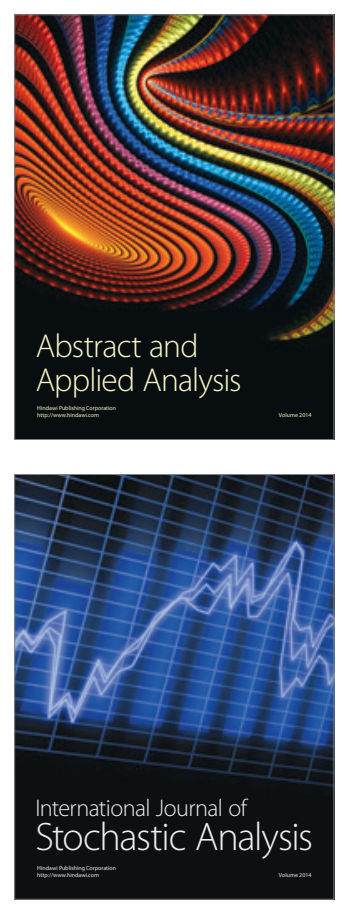

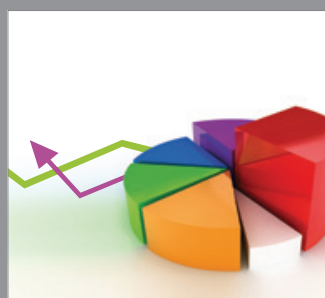

ournal of

Probability and Statistics

Promensencen
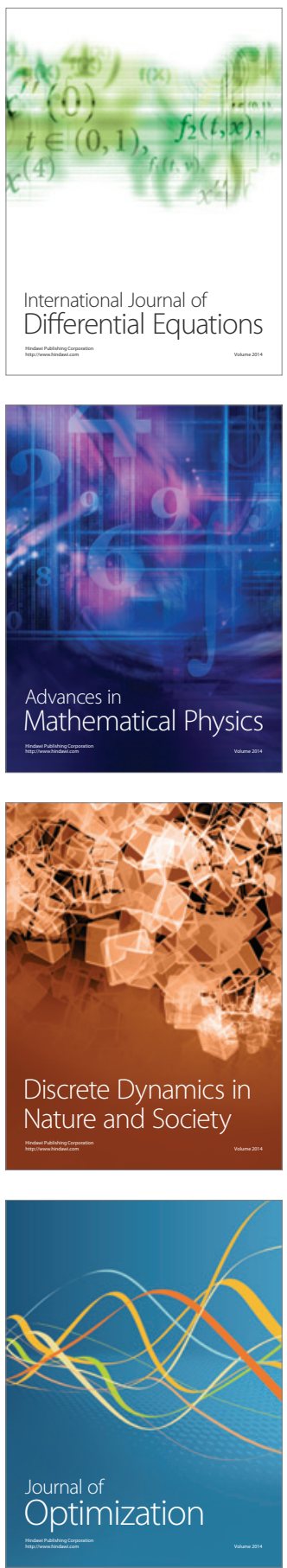